ACCEPTED MANUSCRIPT

\title{
Chitosan/Montmorillonite composite nanospheres for sustained antibiotic delivery at post-implantation bone infection treatment
}

To cite this article before publication: Ceren Kimna et al 2019 Biomed. Mater. in press https://doi.org/10.1088/1748-605X/ab1a04

\section{Manuscript version: Accepted Manuscript}

Accepted Manuscript is "the version of the article accepted for publication including all changes made as a result of the peer review process, and which may also include the addition to the article by IOP Publishing of a header, an article ID, a cover sheet and/or an 'Accepted

Manuscript' watermark, but excluding any other editing, typesetting or other changes made by IOP Publishing and/or its licensors"

This Accepted Manuscript is @ 2018 IOP Publishing Ltd.

During the embargo period (the 12 month period from the publication of the Version of Record of this article), the Accepted Manuscript is fully protected by copyright and cannot be reused or reposted elsewhere.

As the Version of Record of this article is going to be / has been published on a subscription basis, this Accepted Manuscript is available for reuse under a CC BY-NC-ND 3.0 licence after the 12 month embargo period.

After the embargo period, everyone is permitted to use copy and redistribute this article for non-commercial purposes only, provided that they adhere to all the terms of the licence https://creativecommons.org/licences/by-nc-nd/3.0

Although reasonable endeavours have been taken to obtain all necessary permissions from third parties to include their copyrighted content within this article, their full citation and copyright line may not be present in this Accepted Manuscript version. Before using any content from this article, please refer to the Version of Record on IOPscience once published for full citation and copyright details, as permissions will likely be required. All third party content is fully copyright protected, unless specifically stated otherwise in the figure caption in the Version of Record.

View the article online for updates and enhancements. 


\title{
Chitosan/Montmorillonite composite nanospheres for sustained antibiotic delivery at post-implantation bone infection treatment
}

\author{
Ceren Kimna ${ }^{1}$, Sibel Deger ${ }^{1}$, Sedef Tamburaci ${ }^{1,2}$ and Funda Tihminlioglu ${ }^{1}$ \\ ${ }^{1}$ Department of Chemical Engineering, Izmir Institute of Technology, Izmir, Turkey \\ ${ }^{2}$ Graduate Program of Biotechnology and Bioengineering İzmir Institute of Technology, İzmir, Turkey
}

E-mail: fundatihminlioglu@iyte.edu.tr

Received xxxxxx

Accepted for publication $\mathrm{xxxxx}$

Published xxxxxx

\begin{abstract}
Despite the advancements in bone transplantation operations, inflammation is still a serious problem that threatens human health at the post-implantation period. Conventional antibiotic therapy methods may lead to some side effects such as ototoxicity and nephrotoxicity, especially when applied in high doses. Thérefore, local drug delivery systems play a vital role in bone disorders due to the elimination of the disadvantages introduced by conventional methods. In the presented study, it was aimed to develop Vancomycin (VC) and Gentamicin (GC) loaded chitosan-montmorillonite nanoclay composites (CS/MMT) to provide required antibiotic doses to combat post-implantation infection. CS/MMT nanocomposite formation was supplied by microfluidizer homogenization and spherical drug carrier nanoparticles were obtained by electrospraying technique. Three factors; voltage, distance and flowrate were varied to fabricate spherical nanoparticles with uniform size. Emprical model was developed to predict nanosphere size by altering process variables. Nanospheres were characterized in terms of morphology, hydrodynamic size, zeta potential, drug encapsulation efficiency and release profile. Drug loaded nanospheres have been successfully produced with a size range of 180-350 nm. Nanocomposite drug carriers showed high encapsulation efficiency (80-95\%) and prolonged release period when compared to bare chitosan nanospheres. The drug release from nanocomposite carriers was monitored by diffusion mechanism up to 30 days. The in vitro release medium of nanospheres showed strong antimicrobial activity against gram-positive $S$. aureus and gram-negative $E$. coli bacteria. Furthermore, it was found that the nanospheres did not show any cytotoxic effect to fibroblast (NIH/3T3) and osteoblast (SaOS-2) cell lines. The results demonstrated that the prepared composite nanospheres can be a promising option for bone infection prevention at the post implantation period.
\end{abstract}

Keywords: nanocomposite, drug delivery, antibiotic, bone, infection

\section{Introduction}

There has been a significant increase in the bone disorders due to the population aging and the sedentary lifestyle of the people today. Musculoskeletal problems, severe bone tissue degeneration and advanced inflammatory problems cannot be treated with a medication. Consequently, there is an increasing demand for bone replacement materials to ensure adequate support during the healing process and promote new bone growth. However, inflammation is still a drawback of these operations which should immediately be treated to prevent the biofilm formation on the implant surface. To address this issue, local controlled antibiotic releasing systems can be an alternative to conventional methods to 
prevent the inflammation at the bone defects. The sitespecific drug delivery systems supply an effective delivery and prevent the side effects caused by high dosage of the antibiotics such as toxicity and nephrotoxicity ${ }^{1}$.

The material selection to achieve sustained release is a challenging issue. Therefore, a wide range of materials has been used to adjust the release rate regarding the specified criteria for each system. Thus, natural polymer-based nanoparticles can circumvent these issues due to their biocompatibility, biodegradability and the ease in processability. Chitosan (CS) is a natural aminoglycosidic polymer that has been commonly used in drug delivery systems according to its outstanding properties. The active amino groups of positively charged chitosan provide reactive sites for various groups that make it an ideal candidate for carrying any types of drugs. Chitosan-based carrier systems have been evaluated and showed promising results in local antibiotic delivery in forms of the hydrogel, bead or film ${ }^{2,3,4}$. However, it shows rapid degradation profile and high swelling degree. Thus, it requires a modification to improve its stability which is substantial to design a rate-controlled drug delivery. In order to overcome these drawbacks, anionic excipients, crosslinking or composite formation can be preferred to harden the polymer matrix and prolong the drug release period.

Recently, composite formation with nanofillers has attained a great interest according to the superior properties as compared to biopolymer itself. Polymer-clay nanocomposites are the hybrid materials composed of the synergistic effect of the organic polymer matrix and nanoscale organophilic clay fillers. Montmorillonite (MMT) is a smectite group of minerals that is the most commonly used nanofiller in biopolymeric composite systems. Layered structure of the MMT is suitable for reinforcement purposes and provides a tortuous pathway for drug delivery. Studies showed that MMT nano-reinforcement is a promising approach in drug delivery systems in order to overcome obstructions regarding characteristics of biopolymers. Recent literature findings indicated that chitosan/montmorillonite (CS/MMT) nanocomposite systems can ensure improved cationic and anionic drug stability, enhanced drug permeability and extended drug release profile ${ }^{5,6,7,8}$.

Generally conventional homogenization techniques (sonication systems, twin screw extrusion) were used to disperse nanoparticles in polymer matrix. Recently, microfluidization systems have come into prominence as high-pressure homogenization to obtain efficent dispersion in polymer-inorganic of polymer-bioactive component systems. Microfluidizer systems also overcome the limitations of conventional homogenization systems such as limited dispersion level, change in viscosity of biopolymers and possible disruption ultrasonication probe and leakage of metal in polymer solution. The sandwich structure of MMT offers expandable clay layers that give homogenous properties to the composite system if it could be welldispersed in the polymer matrix. Thus, high-pressure microfluidizer (MF) homogenization can be used for dispersion of individual clay platelets in chitosan matrix for nanocomposite formation. However, there are still limited studies regarding the nanocomposite formation via microfluidizer homogenization ${ }^{9,10}$.

Therefore, the aim of this study is to develop a chitosan/montmorillonite composite system for the delivery of Gentamicin (GC) and Vancomycin (VC) antibiotics which are commonly recommended for bone infection treatment. CS/MMT nanocomposite was formed with microfluidization technique and electrosprayed to obtain spherical nanoparticles. The nanofiller dispersion level in a chitosan matrix was determined with X-Ray Diffraction (XRD) patterns. Two-level full factorial design was used to optimize electrospray process variables. The fabricated nanospheres were characterized with Scanning Electron Microscopy (SEM), Atomic Force/Microscopy (AFM), Fourier Transform Infrared Spectroscopy (FT-IR), Dynamic Light Scattering (DLS), zeta potential and encapsulation efficiency studies. In vitro GC and VC release profiles were determined for sub-acute osteomyelitis treatment. The dominant mechanism in the drug delivery was determined with the appropriate kinetic models. The antibacterial activities of nanospheres against $\mathrm{S}$. aureus and $\mathrm{E}$. coli were determined with the agar diffusion method. The in vitro cytotoxicity of the nanospheres were determined with NIH/3T3 and SaOS-2 cell lines.

\section{Materials and Methods}

\subsection{Materials}

High molecular weight chitosan (Sigma-Aldrich, Germany, Cat. No. 419419), organically modified montmorillonite (Cloisite 10A, Southern Clay Products Inc.) and glacial acetic acid (Analytical grade, Merck) were used for solution preparation. Gentamicin (Genta, IE Ulagay) and vancomycin (Anko-L, Mustafa Nevzat) were selected as model antibiotics. Phosphate buffer saline (PBS) solution $(\mathrm{pH}=7.4$, Sigma-Aldrich) was used for DLS, zeta potential and in vitro drug release studies.

The WST-1 assay (Biovision Inc.), Dulbecco's Modified Eagle Medium (DMEM, Capricorn), penicillin and streptomycin antibiotic solution (Capricorn), fetal bovine serum (FBS, Capricorn) were used for the in vitro cytotoxicity determination. 


\subsection{Preparation and Characterization of CS/MMT Nanocomposite Solution}

Briefly, 2\% (w/v) CS was dissolved in 90\% (v/v) acetic acid solution and stirred overnight. MMT nanoclay $(3 \% \mathrm{w} / \mathrm{w}$ chitosan) was dispersed in distilled water, stirred for $24 \mathrm{~h}$ at room temperature and added to polymer solution dropwise, followed by a continuous stirring. The solution was injected to the $\mathrm{Z}$ type interaction chamber of the microfluidizer (Microfluidics LV1, USA) with 10,000 psi, 3 and 5 passes of working conditions to obtain CS/MMT nanocomposite structure.

Homogenized solutions were prefreezed, lyophilized and stored for further characterization studies. Samples were analyzed with XRD analysis (Philips X'PertPro MRD) to determine the dispersion level of clay platelets in polymer matrix. Diffraction patterns were determined at $40 \mathrm{kV}$ using $\mathrm{Cu}-\mathrm{K} \alpha$ radiation $(\lambda=1.54 \AA)$. Intensities were recorded in a range of $2-30^{\circ}(2 \theta)$ with a scanning rate of $0.139^{\circ} / \mathrm{sec}$. The dspacing value that indicates the distance between the nanoclay silicate layers was calculated with the Bragg's law.

The dispersion of MMT layers and the exfoliated structure of platelets in polymer matrix was also observed with STEM analysis. CS/MMT solution was dropped on Cu-grids (UC-A on Lacey, 400 mesh TED PELLA INC.) and dried at ambient conditions before analysis.

The functional groups in CS, MMT and CS/MMT nanocomposite were determined with FT-IR analysis (Shimadzu FTIR-8400S) by KBr pellet technique at 4000 to 400 to $\mathrm{cm}^{-1}$, with a resolution of $4 \mathrm{~cm}^{-1}$.

\subsection{Fabrication of $C S / M M T$ Nanospheres}

Spherical nanoparticles were fabricated with electrospraying method. Prior to drug loading, 2-level-full factorial design was performed to determine the main effect of process parameters and their two- and three-way

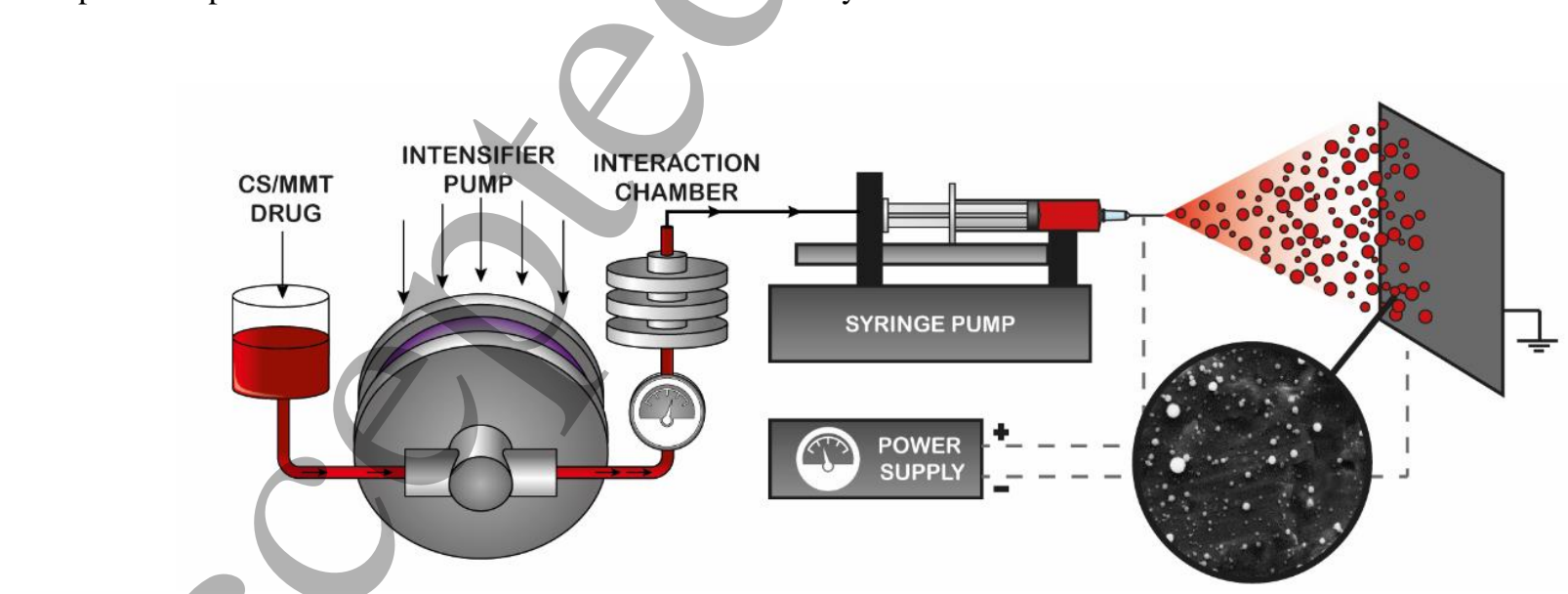

Figure 1. The schematic diagram of microfluidizer and electrospraying unit interactions on the output response (nanosphere diameter) by Minitab $^{\circledR}$ software. Factorial design was selected due to minimize the total number of experiments to achieve the most precise overall optimization ${ }^{11}$. The effect of process parameters was investigated by altering the voltage (10 and $20 \mathrm{kV})$, distance $(5$ and $10 \mathrm{~cm}$ ) and the flowrate of the polymeric solution $(5$ and $10 \mathrm{~mL} / \mathrm{h})$. Needle diameter $(1.27$ $\mathrm{mm})$ and the polymer concentration $(2 \% \mathrm{w} / \mathrm{v})$ were kept constant in order to avoid fiber formation. The acetic acid concentration of the solution was kept constant at $90 \%$ (v/v) since this concentration was found as optimal to decrease the surface tension of the chitosan solution and increases the charge density of jet ${ }^{12}$. The collector surface was covered with aluminum foil and the product was collected on surface. Experiments were randomly replicated as thrice. Then, the morphology and the size of fabricated nanospheres were analyzed by SEM analysis. In each sample, the diameter of minimum 250 nanospheres was measured by ImageJ software to determine the average size.

After optimizing the electrospraying parameters, Vancomycin (VC) and Gentamicin (GC) were added to CS/MMT solutions before microfluidizer homogenization with a polymer to drug weight ratio (P:D) of 4:1, 8:1 and 10:1, 20:1 for VC) and GC antibiotics, respectively. Drug incorporated solutions were homogenized with MF under working conditions of 10,000 psi pressure and 5 passes according to nanocomposite characterization tests. The microfluidized solutions were electrosprayed with a voltage of $20 \mathrm{kV}$, a distance between syringe and collector of $10 \mathrm{~cm}$ and $5 \mathrm{~mL} / \mathrm{h}$ flowrate to fabricate spherical nanoparticles. Figure 1 shows the schematic diagram of homogenization and electrospraying process.

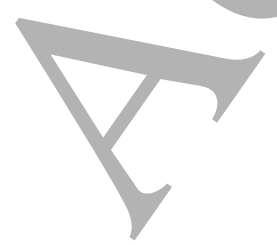




\subsection{Characterization of drug loaded CS/MMT nanospheres}

SEM analysis was performed in order to observe the morphology of the nanospheres with Quanta FEG 250 (at $7 \times 10^{-2}$ mbar and $15 \mathrm{~mA}$ ). Before the analysis, samples were coated with a thin gold layer under argon gas (Emitech K550X). The size distribution of the nanospheres was determined with the Dynamic Light Scattering (DLS) analysis. The samples were suspended in $5 \mathrm{~mL}$ of $1 \mathrm{x}$ phosphate buffer saline (PBS) and $\mathrm{pH}$ was adjusted to 7.4 at $25^{\circ} \mathrm{C}$, sonicated for 5 minutes to prevent aggregation, decanted into a glass scintillation vial and analyzed by Malvern Zetasizer Nano-Zs. The particle size distribution and mean hydrodynamic diameters were analyzed as intensity averaged distributions. The experiments were conducted thrice, and the results were given as the average of 3 runs and 100 scans for each run.

The zeta potential of the nanospheres were analyzed with Malvern Zetasizer Nano-Zs. Samples $(n=3)$ were analyzed in $1 \mathrm{x}$ PBS $(\mathrm{pH}=7.4)$ and ultrapure water at $25^{\circ} \mathrm{C}$. Samples were dispersed in liquid media, vortexed and analyzed with 3 runs and 100 scans per one run. The point of zero charge (PZC) of MMT nanoclay dispersion in 1x PBS was determined by altering the $\mathrm{pH}$ between 3 and 12. The $\mathrm{pH}$ of the nanoclay dispersion was adjusted by using buffer solutions of $0.1 \mathrm{M}$ $\mathrm{HCl}$ and $0.1 \mathrm{M} \mathrm{NaOH}$.

The chemical interactions between drugs and the nanocomposite matrix were determined with FT-IR (Shimadzu FTIR-8400S) analysis at 4000 to 400 to $\mathrm{cm}^{-1}$, with a resolution of $4 \mathrm{~cm}^{-1}$.

In order to calculate the encapsulation efficiency, nanospheres were dissolved in $5 \mathrm{~mL}$ of $1 \mathrm{x}$ PBS $(\mathrm{pH}=7.4)$ and disrupted in an ultrasonic bath for 30 minutes. The solution was filtered, and the amount of entrapped drug was determined by UV-Vis spectroscopy (Varioskan) according to the previously determined calibration curve. The absorbances of the samples were measured at 256 and 282 $\mathrm{nm}$ for GC and VC, respectively. Encapsulation efficiency of was calculated according to the equation below (Equation 1): $E E(\%)=($ Amount of drug/Theoretical amount of drug) $x 100$

\subsection{In vitro drug release}

In vitro $\mathrm{VC}$ and $\mathrm{GC}$ release from $\mathrm{CS}$ and $\mathrm{CS} / \mathrm{MMT}$ nanocomposites was determined under immersion conditions. Samples $(n=3)$ were placed in $1 \mathrm{x}$ PBS $(\mathrm{pH}=7.4)$ and incubated in an orbital shaker at $75 \mathrm{rpm}$ and constant temperature of $37^{\circ} \mathrm{C}$. Release media were collected at predetermined time intervals and replaced with fresh PBS. The volume of the incubation medium was kept constant during the analysis. The release rate was determined with absorbance data taken by UV-VIS spectroscopy (Varioskan).
Furthermore, the drug release profiles were fitted to the Firstorder, Higuchi, Hixson-Crowell and Baker-Lonsdale kinetic models. Then, Korsemeyer-Peppas model was applied to understand the underlying mechanism in release of antibiotics $^{13}$.

\subsection{Antimicrobial activity}

Gram-positive Staphylococcus aureus (RSKK 1009) and gram-negative Escherichia coli (ATCC® 25922) were used as model organisms for antimicrobial activity tests. The frozen bacteria stocks were activated at $37^{\circ} \mathrm{C}$ overnight prior to experiments. $3.4 \times 10^{7} \mathrm{cfu} \mathrm{mL}^{-1}$ of early mid-log phase culture of $S$. aureus in tryptic soy broth and $2.5 \times 10^{7} \mathrm{cfu} \mathrm{mL}^{-1}$ of $E$. coli in nutrient agar were incubated at $37^{\circ} \mathrm{C}$ for $24 \mathrm{~h}$.

Firstly, the bacteria cultures were incubated with serially diluted antibiotic solutions for $24 \mathrm{~h}$ of incubation in 6-well plates in an orbital shaker at $55 \mathrm{rpm}$ and $37^{\circ} \mathrm{C}$ to observe whether the concentration of released antibiotic is sufficient to prevent the bacterial colonization. Then, the bacteria solution containing antibiotics were placed on agar and incubated at $37^{\circ} \mathrm{C}$ for $24 \mathrm{~h}$. The minimum required drug concentration was determined by observation of bacteria colonization on petri dishes.

The antimicrobial activity of drug release media of CS/MMT nanospheres was evaluated with the agar diffusion method. The antimicrobial susceptibility discs (Oxoid ${ }^{\mathrm{TM}}$ ) were gently put on the surface of inoculated agar with bacteria. $10 \mu \mathrm{l}$ of drug release media taken at specified time intervals ( 6 hours, 24 hours and 25 days) were dropped on discs $(n=3)$. Vancomycin and amoxicillin antibiotic discs were used as positive; blank disk was used as a negative control. Petri dishes were incubated at $37^{\circ} \mathrm{C}$ overnight and the clear zones around the discs were recorded after 1 day of incubation.

\subsection{Statistical analysis}

The significance of the differences between the results was evaluated by one-way and two-way analysis of variance (ANOVA) with $\mathrm{P}<0.05$ as a minimum level of significance. The statistical analysis was performed using GraphPad Prism 7.0 (GraphPad Software Inc.). All experiments were conducted as three replicates and the results were expressed as a mean \pm standard deviation.

\section{Results}

\subsection{Nanocomposite Characterization}

XRD study was performed to display the clay distribution level in the polymer matrix. Figure 3 depicts the XRD pattern of the chitosan (CS), montmorillonite (MMT) and CS/MMT composites homogenized with 10,000 psi pressure and pass number of 3 and 5 . The characteristic peak was

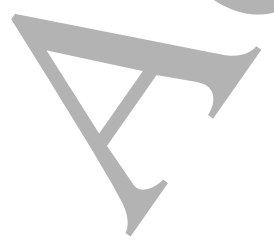


depicted in $2 \theta=20.7^{\circ}$ due to diffraction of chitosan powder, which well agrees with the previous reports ${ }^{14}$. In a study, it was observed that the characteristic peak for pristine nanoclay at $2 \theta=7^{\circ}$ shifted to small angles with increasing the chitosan content due to intercalation ${ }^{8}$. Similarly, the diffraction peak of MMT (Cloisite 10A) was observed approximately in $2 \theta=7.23^{\circ}$. According to the Bragg's law, the peak at $7.23^{\circ}$ indicates a basal d-spacing of $12.22 \AA$. This peak has shifted left to $6.78^{\circ}$ with a microfluidizer application of 10,000 psi and 3 passes due to the intercalation of MMT layers. It was calculated that the $\mathrm{d}$-spacing value increased to $13.03 \AA$, which is a sign of an intercalated structure ${ }^{15}$. The permeation of chitosan into MMT galleries broadened the layer spacing. Two hydroxyls and one amino functional group of chitosan can provide an interaction between hydroxyl edges of MMT layers by hydrogen bonding to ensure the penetration into MMT nanosheets ${ }^{16}$. Cyclic microfluidizer application with 5 pass at 10,000 psi caused exfoliation of the single clay layers in the polymer matrix. Furthermore, the characteristic peak of MMT could not be observed in $2 \theta$ range of $3-9^{\circ}$ at 5 passes of MF application that indicates the exfoliated structure ${ }^{17}$. STEM images also showed that MMT was effectively dispersed in polymer matrix. Layered structures of MMT were observed clearly with 25000x, 50000x, 100000x and 200000x magnifications (Figure 2 a,b,c,d). MMT particles were dispersed as both intercalated and exfoliated structures in chitosan matrix. MMT particle size range was obtained between 180-400 nm in thickness and 700-1300 nm in width. Therefore, microfluidizer working conditions were determined as 10,000 psi and 5 passes to enrich exfoliated structure for antibiotic loaded nanospheres to achieve sustained drug release.
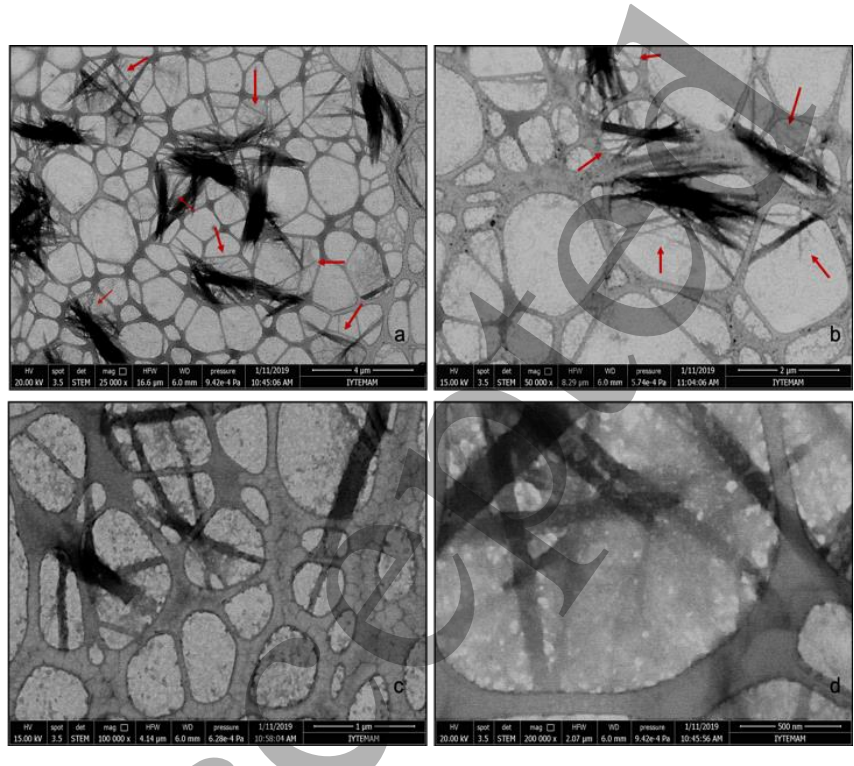

Figure 2. STEM images of CS/MMT nanocomposite

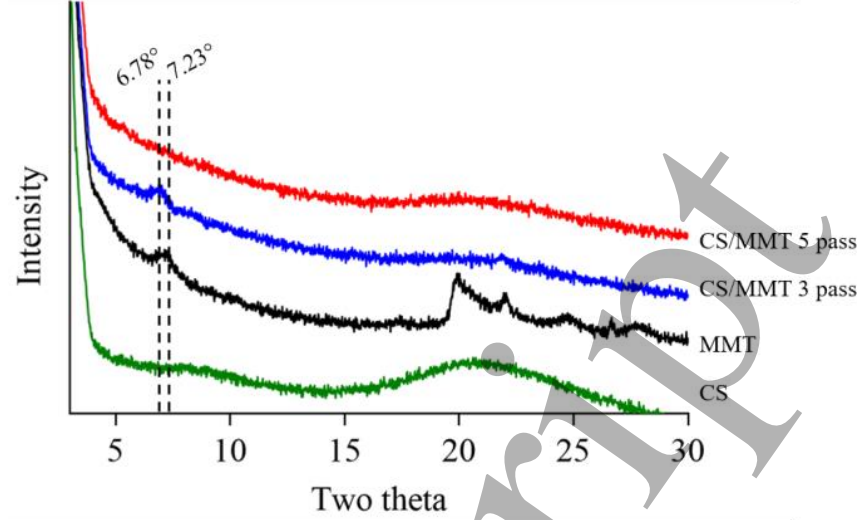

Figure 3. XRD spectra of chitosan (CS), montmorillonite (MMT) and microfluidized CS/MMT nanocomposites

FT-IR analysis was performed to determine the functional groups of chitosan, MMT nanoclay and CS/MMT nanocomposites (Figure 4). The FT-IR spectrum of chitosan consists of characteristic bands appeared at $1655 \mathrm{~cm}^{-1}(\mathrm{C}=\mathrm{O}$ stretching of amide I), $1560 \mathrm{~cm}^{-1}$ (N-H bending of amine) and $1380 \mathrm{~cm}^{-1}$ (C-N stretching and $\mathrm{N}-\mathrm{H}$ bending of amide linkages). The main characteristic peaks of MMT are at 3667 $\mathrm{cm}^{-1}$ (Al-OH stretching), $1020 \mathrm{~cm}^{-1}$ (Si-O stretching), 913 $\mathrm{cm}^{-1}$ (Al-Al-OH). The peaks of number 5 (-CH- stretching) and $6(-\mathrm{CH} 2$ vibration) were located on MMT spectra due to the organic modification ${ }^{18}$. In the case of CS/MMT composite groups, the IR spectra showed that the characteristic bands of both CS and MMT that appeared in the CS/MMT composite. The characteristic amide I (1655 $\left.\mathrm{cm}^{-1}\right)$, amine $\left(1560 \mathrm{~cm}^{-1}\right)$ and amide III $\left(1380 \mathrm{~cm}^{-1}\right)$ peaks of chitosan slightly shifted to left in CS/MMT composite. The characteristic band of MMT due to -CH2 vibration $\left(2916 \mathrm{~cm}^{-}\right.$ 1) was seen in the CS/MMT composite. The interaction of CS and MMT was ensured by the hydrogen bonding due to hydroxylated silicates of MMT and, amino and hydroxyl groups of $\mathrm{CS}^{15}$. The MMT Si-O stretching $\left(1020 \mathrm{~cm}^{-1}\right)$ was overlapped with CS peaks in the IR spectra of CS/MMT group. The peak at $2990 \mathrm{~cm}^{-1}$ due to $\mathrm{OH}$ stretching was shifted to $2934 \mathrm{~cm}^{-1}$ and overlapped with the characteristic peak of MMT due to -CH2- vibration at $2916 \mathrm{~cm}^{-1}$. The intensities of the characteristic peak of MMT due to Al-OH$\mathrm{Al}$ stretching $\left(913 \mathrm{~cm}^{-1}\right)$ and -CH- bending $\left(2852 \mathrm{~cm}^{-1}\right)$ were decreased in composite groups when compared to pure MMT. This fact also confirms the interaction between chitosan and $\mathrm{MMT}^{19}$. 


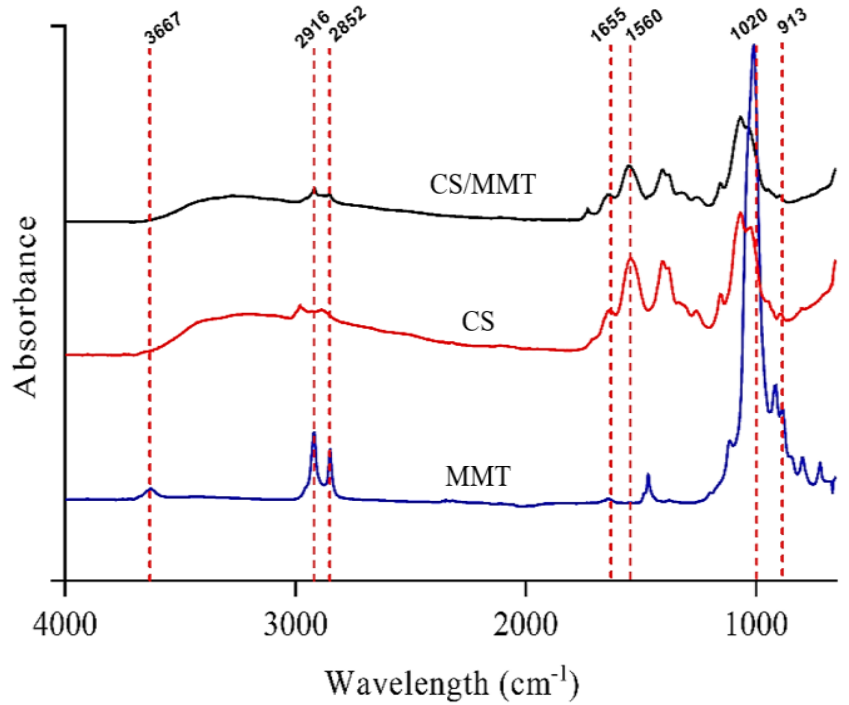

Figure 4. IR spectrum of chitosan, MMT and CS/MMT composite groups

\subsection{Optimization on Electrospray Process Parameters}

The effect of electrospray parameters on nanosphere formation were investigated by measuring the nanosphere sizes from images taken by SEM analysis (Figure 6).

The main and interactive effects of experimentally controlled factors like voltage, distance and flowrate on the response (particle diameter) were investigated through the $2^{3}$ two-level full factorial design. The independent variables and the ranges were tabulated in Table 1 . The size of nanosphere samples in each run was given in Table 2 .

The experimental results were analysed with Minitab software and the main effects on average nanosphere diameter were shown in Figure 5.a. Results implied that smaller nanosphere size could be achieved with higher voltage, higher distance and lower flowrate. It was inferred that each main effect significantly affects the response (Figure 5.b).

Table 1. Factors and levels for the electrospraying process

\begin{tabular}{|l|l|l|l|}
\hline \multirow{2}{*}{$\begin{array}{l}\text { Independent } \\
\text { variable }\end{array}$} & \multirow{2}{*}{$\begin{array}{l}\text { Coded } \\
\text { Symbol }\end{array}$} & \multicolumn{2}{|l|}{ Range and leyel } \\
\cline { 3 - 4 } & Low $(-1)$ & High $(+1)$ \\
\hline Voltage $(\mathrm{kV})$ & A & 10 & 20 \\
\hline Distance $(\mathrm{cm})$ & B & 5 & 10 \\
\hline Flowrate $(\mathrm{mL} / \mathrm{h})$ & $\mathrm{C}$ & 5 & 10 \\
\hline
\end{tabular}

Table 2. Complete design layout of 3 factors, 2-level full factorial design and the response obtained from randomly performed experiments

\begin{tabular}{|l|l|l|l|l|l|l|}
\hline \multirow{2}{*}{$\begin{array}{l}\text { Std. } \\
\text { order }\end{array}$} & $\begin{array}{l}\text { Run } \\
\text { order }\end{array}$ & \multirow{2}{*}{$\begin{array}{l}\text { Group } \\
\text { codes }\end{array}$} & $\begin{array}{l}\text { laded } \\
\text { values of } \\
\text { independent } \\
\text { variables }\end{array}$ & $\begin{array}{l}\text { Response } \\
\text { Nanosphere } \\
\text { diameter(nm) }\end{array}$ \\
\cline { 3 - 6 } & & & A & B & C & \\
\hline 1 & 4 & A & - & - & - & $127.04 \pm 7.7$ \\
\hline 2 & 6 & B & - & - & + & $141.75 \pm 4.0$ \\
\hline 3 & 3 & C & - & + & - & $87.67 \pm 2.1$ \\
\hline 4 & 5 & D & - & + & + & $104.73 \pm 5.1$ \\
\hline 5 & 7 & E & + & - & - & $111.75 \pm 4.7$ \\
\hline 6 & 1 & F & + & - & + & $116.91 \pm 2.0$ \\
\hline 7 & 8 & G & + & + & - & $86.67 \pm 3.5$ \\
\hline 8 & 2 & H & + & + & + & $96.45 \pm 4.0$ \\
\hline
\end{tabular}

Therefore, ANOVA was done with 95\% two-sided confidence interval to evaluate the significance of the effects and the interactions among the variables. It was observed that besides main/effects, two-way interactions of voltagedistance and voltage-flowrate also affected the size of nanospheres. However, two-way interactions of distance and flowrate, and the three-way interaction were not statistically significant. The analysis of variance for nanosphere diameters with significant terms is given in Table 3 .

The uncoded mathematical model for $2^{3}$ factorial design after discarding insignificant terms can be represented as:

$D(n m)=171.4-2.667 A-10.77 B+4.97 C+0.3133 A B-0.18 A C$

where $\mathrm{D}$ is diameter of the nanospheres. The factorial regression model showed a high regression coefficient $\left(R^{2}\right)$ in the determined range as 0.958 .

As a result of the statistical design of the electrospray parametric optimization experiments followed by regression, electrospray parameters were set as a voltage of $20 \mathrm{kV}$, a distance of $10 \mathrm{~cm}$ and a flowrate of $5 \mathrm{~mL} / \mathrm{h}$ that results with uniformly distributed nanospheres with the smallest size and spherical shape. 


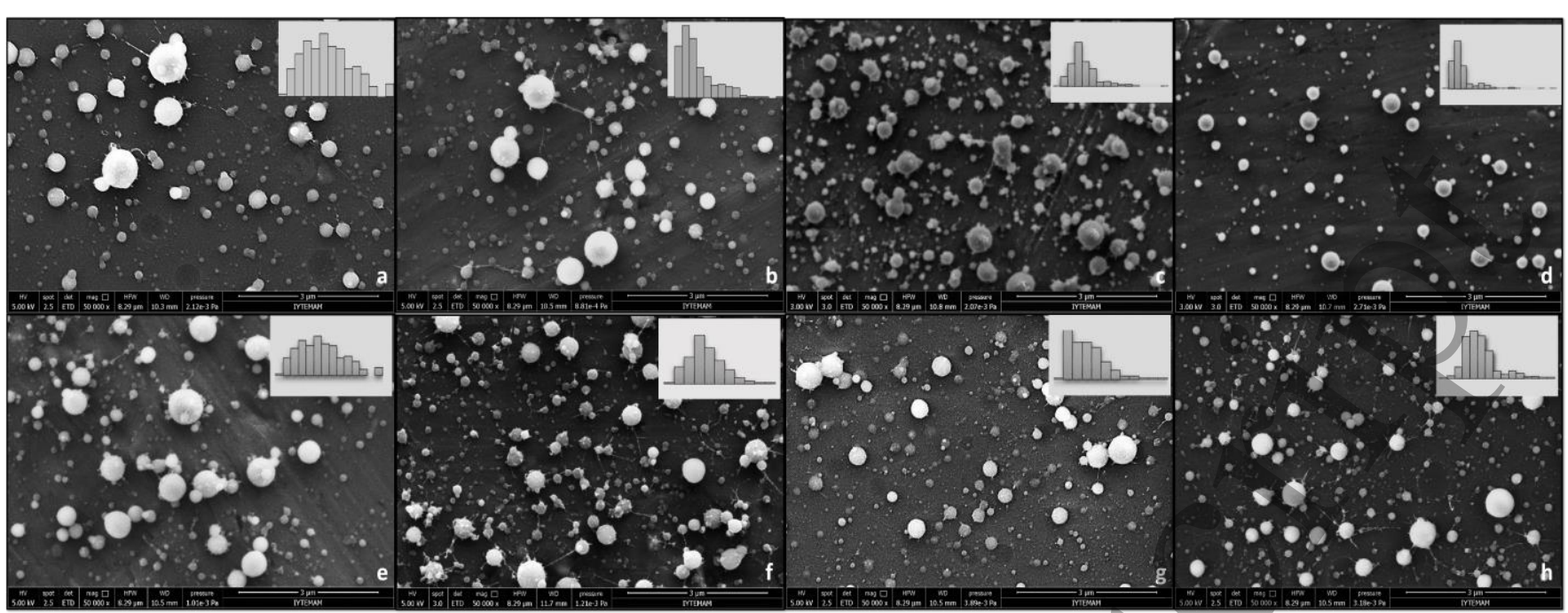

Figure 6. Electrosprayed chitosan particles (Groups codes were depicted in Table 2.)

Table 3. Analysis of variance for nanosphere diameter

\begin{tabular}{|l|l|l|l|l|l|}
\hline Source & Df & Sum of squares & Mean square & F value & p-value \\
\hline Model & 5 & 7743.6 & 1548.73 & 81.95 & $<0.0001$ \\
\hline Voltage & 1 & 962.67 & 962.67 & 50.94 & $<0.0001$ \\
\hline Distance & 1 & 5520.67 & 5520.67 & 292.13 & $<0.0001$ \\
\hline Flowrate & 1 & 770.67 & 770.67 & 40.78 & $<0.0001$ \\
\hline Voltage*Distance & 1 & 368.17 & 368.17 & 19.48 & $<0.0001$ \\
\hline Voltage*Flowrate & 1 & 121.50 & 121.50 & 6.43 & 0.021 \\
\hline Residual & 18 & 340.17 & 18.90 & & \\
\hline Total & 23 & 8083.83 & & & \\
\hline
\end{tabular}




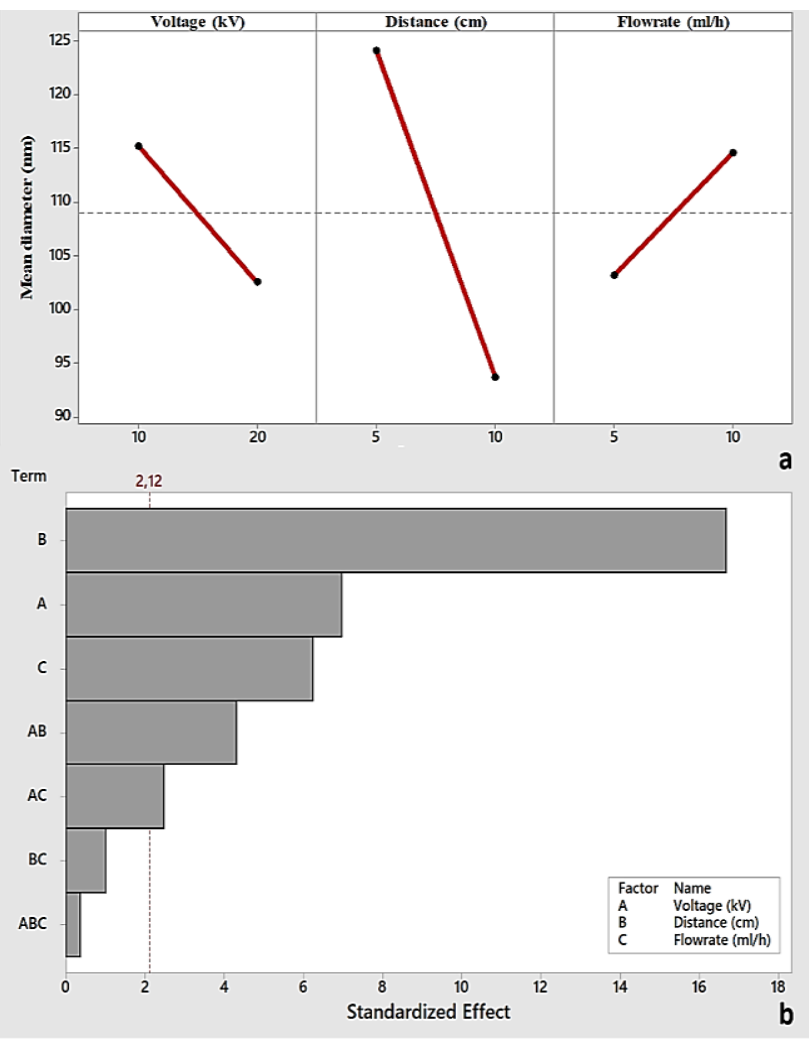

Figure 5. Main effects for nanospheres diameter

(a); Pareto chart for standardized effects $(\alpha=0.05)$ (b).

\subsection{Characterization of the VC and GC Loaded CS/MMT Nanospheres}

According to the SEM images and hydrodynamic size distributions, it can be observed that selected electrospray parameters were suitable for nanosphere formation since the formed nanoparticles showed a smooth surface and perfectly spherical shape (Figure 7). The size distribution of nanospheres dispersed in 1x PBS ( $\mathrm{pH}=7.4)$ was tabulated and given by intensity \% (Table 4 ). Low polydispersity index (PDI) values $(<0.2)$ shows the homogeneity of the system with a narrow size distribution. The average hydrodynamic size of CS/MMT nanospheres was found as $65.2 \pm 4.3 \mathrm{~nm}$. DLS results indicated that hydrodynamic size increases with drug incorporation when compared to the control, i.e. unloaded, CS/MMT nanospheres. The mean hydrodynamic size was found as $351 \pm 20.18 \mathrm{~nm}$ and $210 \pm 19.25 \mathrm{~nm}$ for the groups having chitosan to Vancomycin ratio of $4: 1$ and $8: 1$, respectively. The mean hydrodynamic sizes of Gentamicin loaded nanospheres were found as $246.4 \mathrm{~nm}$ and $181.6 \mathrm{~nm}$ for the groups having P:D of 10:1 and 20:1, respectively.

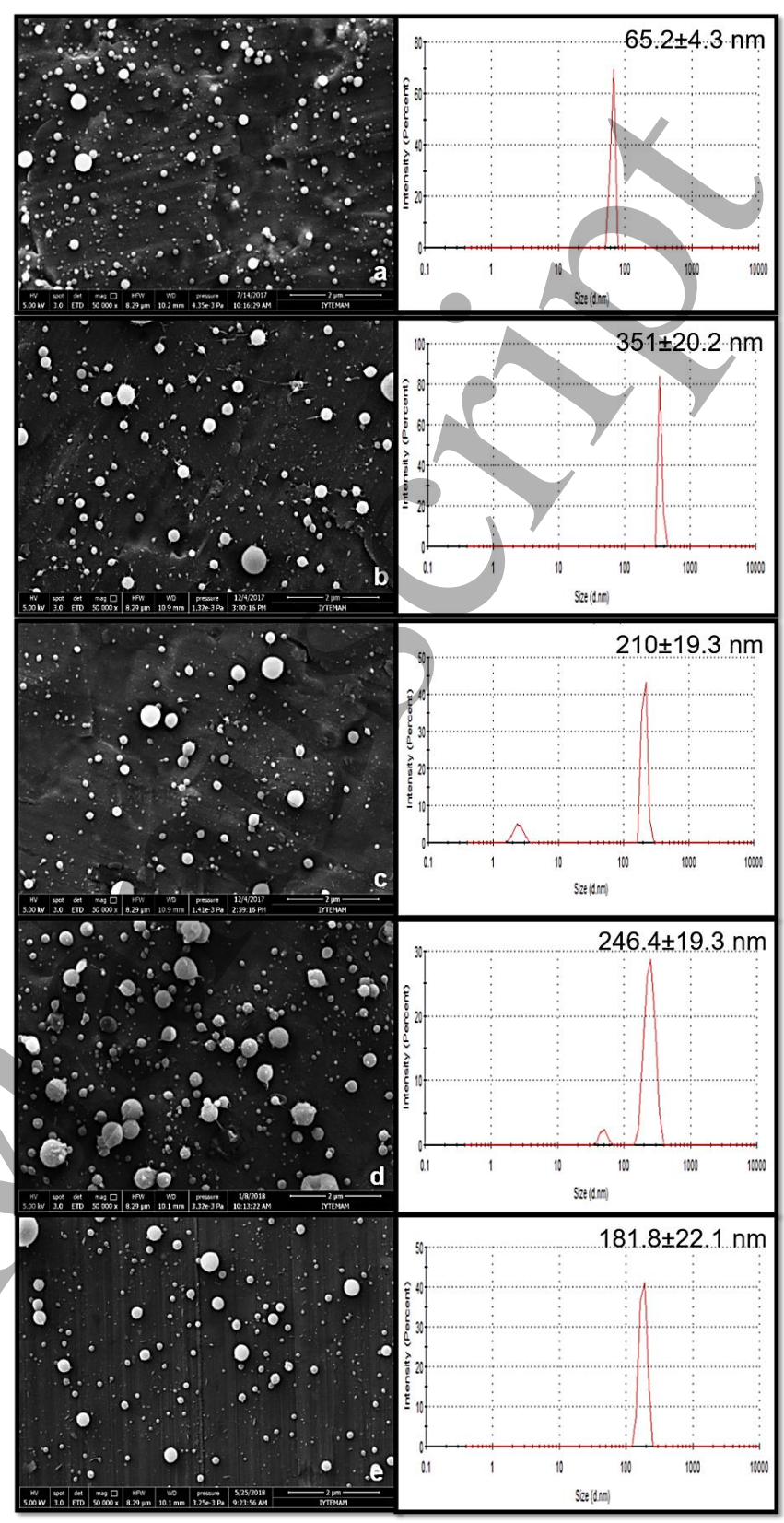

Figure 7. SEM images and intensity based hydrodynamic size distributions of CS/MMT (a), CS/MMT-VC (4:1) (b), CS/MMT-VC (8:1) (c), CS/MMT-GC (10:1) (d) and CS/MMT-GC(20:1) (e) nanospheres (Scale bars represent 2 $\mu \mathrm{m})$.

The point of zero charge (PZT) of MMT nanoclay was found in $\mathrm{pH} 6.2$ (Figure 8). In literature, it was indicated that the PZC of MMT varies between $6.4-7.2^{20}$. The zeta potential of MMT varied at different $\mathrm{pH}$ values due to the proton adsorption or desorption on the edge - $\mathrm{OH}$ - groups and isomorphous substitutions at the faces of platelets ${ }^{21}$. The amphoteric parts were positively or negatively charged due to the $\mathrm{pH}$ of the media. Some parts of cations do not strongly 
hold to the structure hence readily exchanged by other ions. Therefore, it can be inferred that particle stability is assured by homogeneous dispersion of negatively charged MMT in positively charged $\mathrm{CS}$ matrix in the $\mathrm{pH}$ of the body fluid.

Moreover, the zeta potential of nanospheres was measured immediately after suspended in ultrapure water and PBS (Table 4). The zeta potential of CS/MMT nanospheres were found higher in $\mathrm{ddH}_{2} \mathrm{O}$ when compared to PBS. The continuous dissolution of atmospheric $\mathrm{CO}_{2}$ lead to $\mathrm{pH}$ reduction in water that paves the way for protonation of amine groups of chitosan ${ }^{22}$. Therefore, relatively positive zeta potential values were observed. It was found that prepared CS/MMT nanospheres are positively charged due to the polycationic nature of chitosan. The zeta potential of neat CS nanospheres was found in a range of 24-29 $\mathrm{mV}$. However, the zeta potential of neat CS/MMT nanospheres was measured as 13.3-18.7 $\mathrm{mV}$ due to the negative charge of MMT nanoclay in the working $\mathrm{pH}$. Hence, prepared drug loaded nanospheres carried their stability in aqueous media according to their repulsive forces.

All antibiotic loaded nanospheres showed a positive zeta potential that indicates electric repulsion preventing aggregate formation. In addition, positive surface charge is advantageous in antibiotic delivery systems since most of the bacterial cellular membrane surfaces are negatively charged $^{23}$.

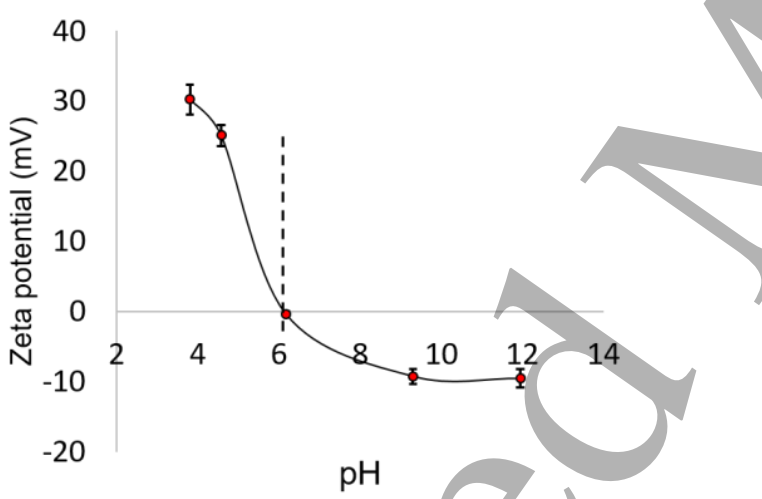

Figure 8 . The zeta potential of MMT in various $\mathrm{pH}$

Additionally, chemical interactions between the antibiotics and CS/MMT nanocomposite were analyzed with FT-IR analysis. Figure 9.a depicts the IR spectra of the CS/MMT, Vancomycin and the Vancomycin-loaded CS/MMT groups. Vancomycin has a characteristic absorption band of $-\mathrm{OH}-$ stretching $\left(3450 \mathrm{~cm}^{-1}\right), \quad \mathrm{C}=\mathrm{O}$ stretching $\left(1652 \mathrm{~cm}^{-1}\right)$, aromatic $\mathrm{C}=\mathrm{C}$ stretching $\left(1502 \mathrm{~cm}^{-1}\right)$ and phenolic groups $\left(1232 \mathrm{~cm}^{-1}\right)^{24,25}$. The existence of most of these bands confirmed the presence of Vancomycin in the nanocomposite spheres.
The interaction between the polymer matrix and Vancomycin was supported with hydrogen bonds (3750$2250 \mathrm{~cm}^{-1}$ ) and observed as a shifted and broadened band.

The FT-IR spectra of CS/MMT, Gentamicin and Gentamicin-loaded CS/MMT groups with a polymer to drug ratio of 10:1 and 20:1 were depicted in Figure 9.b. In literature, it was indicated that the Gentamicin spectra includes a strong absorption band due to stretching vibrations of C-N and C-O between 1300-900 $\mathrm{cm}^{-1} ; \mathrm{N}-\mathrm{H}$ vibration at $1650-1400 \mathrm{~cm}^{-1}$ 26. Most of the characteristic peaks of Gentamicin were depicted in the IR spectra of Gentamicin incorporated groups. The Gentamicin incorporation into CS/MMT matrix can be explained by hydrogen bonding due to shifts of amide I and II groups of the CS. The intensity of the hydroxyl peak $\left(3420 \mathrm{~cm}^{-1}\right)$ was weakened and broadened in Gentamicin loaded CS/MMT matrix. This change was linked with the merge of chitosan and gentamicin -OHbending at $3415 \mathrm{~cm}^{-122,2}$

The structure of the carrier matrix and the polymer to drug ratio (P:D) are known as significant factors affecting the drug release rate and the drug encapsulation efficiency (EE\%) of the polymeric carrier material. It was observed that the nanocomposite formation significantly increased the encapsulation efficiency (Table 5). The nanocomposite structure improved the Vancomycin entrapment of the matrix from 80 to $86 \%$ without changing the P:D ratio. Similarly, Gentamicin incorporation was significantly increased from 76 to $94 \%$ by aid of exfoliated nanocomposite matrix. The entrapment efficiency of Vancomycin loaded groups were found as 79.04 and $86.56 \%$ for the P:D of $4: 1$ and $8: 1$, respectively. The incorporation of the drug into the polymer matrix becomes easier as P:D increases, which results in higher entrapment efficiency value. Similar findings were also observed in the literature regarding polymeric nanoparticulate drug delivery systems ${ }^{27,28}$. Similarly, the entrapment efficiencies of Gentamicin loaded groups were found as 90.59 and $94.73 \%$ for the P:D of 10:1 and 20:1, respectively.

Table 3. Encapsulation efficiencies of drug loaded nanospheres

\begin{tabular}{|c|c|c|c|}
\hline Material & Drug & Polymer:Drug & $\begin{array}{l}\text { Encapsulation } \\
\text { efficiency }(\%)\end{array}$ \\
\hline $\mathrm{CS}$ & \multirow{3}{*}{$\mathrm{VC}$} & $8: 1$ & $80 \pm 3$ \\
\hline CS/MMT & & $4: 1$ & $79 \pm 1$ \\
\hline CS/MMT & & $8: 1$ & $87 \pm 3$ \\
\hline $\mathrm{CS}$ & \multirow{3}{*}{$\mathrm{GC}$} & $20: 1$ & $76 \pm 2$ \\
\hline CS/MMT & & $10: 1$ & $91 \pm 5$ \\
\hline CS/MMT & & $20: 1$ & $95 \pm 4$ \\
\hline
\end{tabular}


Table 4. Zeta potential and hydrodynamic size distributions of nanospheres

\begin{tabular}{|c|c|c|c|c|}
\hline \multirow{2}{*}{ Groups } & \multicolumn{2}{|c|}{ Zeta Potential $(\mathrm{mV})$} & \multicolumn{2}{|c|}{ DLS Measurements (in PBS) } \\
\hline & in PBS & in $\mathrm{ddH}_{2} \mathrm{O}$ & Average size (nm) & PDI \\
\hline CS/MMT & $13.3 \pm 1.26$ & $18.7 \pm 0.06$ & $65.2 \pm 4.3$ & 0.09 \\
\hline CS/MMT-VC (4-1) & $5.6 \pm 0.21$ & $8.2 \pm 1.12$ & $351 \pm 20.2$ & 0.11 \\
\hline CS/MMT-VC (8-1) & $12.3 \pm 1.72$ & $17.4 \pm 2.30$ & $210 \pm 19.3$ & 0.16 \\
\hline CS/MMT-GC (10-1) & $4.3 \pm 0.03$ & $7.8 \pm 1.48$ & $246.4 \pm 19.3$ & \\
\hline CS/MMT-GC (20-1) & $6.9 \pm 0.55$ & $9.9 \pm 0.78$ & $181.8 \pm 22.1$ & \\
\hline
\end{tabular}
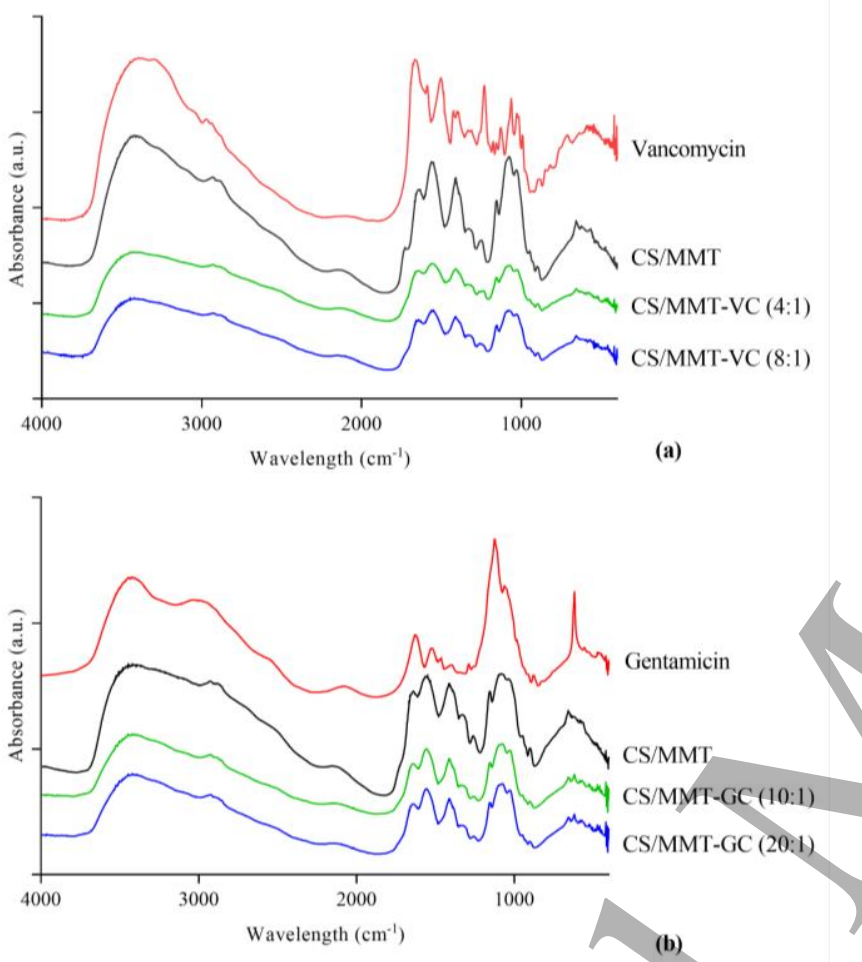

Figure 9. IR spectrum of Vancomycin (a) and Gentamicin (b) loaded nanospheres.

\subsection{In vitro drug release}

The in vitro Vancomycin and Gentamicin release over time were identified to evaluate the feasibility of CS/MMT nanocomposites as a long-term local drug delivery system. In literature, it was observed that incorporation of layered clay minerals in chitosan coating on titanium implants can prolong the release of Vancomycin from few days to month and decrease the burst release ${ }^{29}$.

The cumulative drug release profiles of Vancomycin and Gentamicin drugs with two different P:D ratio was given in Figures $10 \mathrm{a}, \mathrm{b}$ respectively. As seen in the cumulative plots, two-step release profile was observed as an initial burst and sustained release in nanocomposite groups.

In the control group, i.e. without MMT nanofiller addition, the release could only be tracked for few hours. In order to increase the stability of the chitosan nanocarriers, here we have examined the effect of MMT nanofiller in the chitosan matrix as a nanocomposite structure. The Vancomycin release in neat CS nanospheres could only be monitored for 4 hours of incubation with a burst release profile about $100 \%$ whereas the drug release was sustained up to 30 days with a nanocomposite structure.

In composite groups, the burst release in 6 hours was followed by a controlled release profile. $38 \pm 1.86 \%$ of Vancomycin has released from CS/MMT-VC (4:1) nanospheres in first 6 hours, followed by a controlled release of $88 \pm 1.53 \%$ of the total amount of drug until it reaches a plateau. Finally, $96 \pm 1.15 \%$ of the Vancomycin was released to the incubation media. It was observed that nanospheres containing less amount of Vancomycin (CS/MMT-VC (8:1)) showed a slower release rate when compared to CS/MMTVC $(4: 1)$ group. $24 \pm 0.58 \%$ of GC release in 6 hours is followed by a controlled release. Finally, $96 \pm 1.15 \%$ of the Vancomycin released to the incubation media.

By evaluating the release pattern shown in Figure 9, neat CS nanocarriers could entrap the hydrophilic Gentamicin only for 4 hours. On the other hand, the release was prolonged up to 30 days with the nanocomposite structure. 84 and $88 \%$ of the drug released from CS/MMT-GS (20:1) and CS/MMT-GS (10:1) nanospheres in 30 days, respectively. Similarly, the decrease in release rate was observed when P:D ratio was increased. CS/MMT nanospheres that contain less amount of drug decelerated the burst release effect by entrapping the drug stronger inside the polymer matrix. Relatively linear trend was observed for CS/MMT-GC 10:1 group after the burst release until it reaches the plateau on the $20^{\text {th }}$ day. CS/MMT-GC (20:1) group showed a biphasic trend due to possible degradation of nanospheres at day 14 .

It was reported that the minimum Gentamicin concentration should be in $1-10 \mathrm{ng} / \mathrm{mL}$ at the defect site to promote bone healing ${ }^{30}$. The results showed that the therapeutic drug level was achieved without exceeding the toxic concentration. 


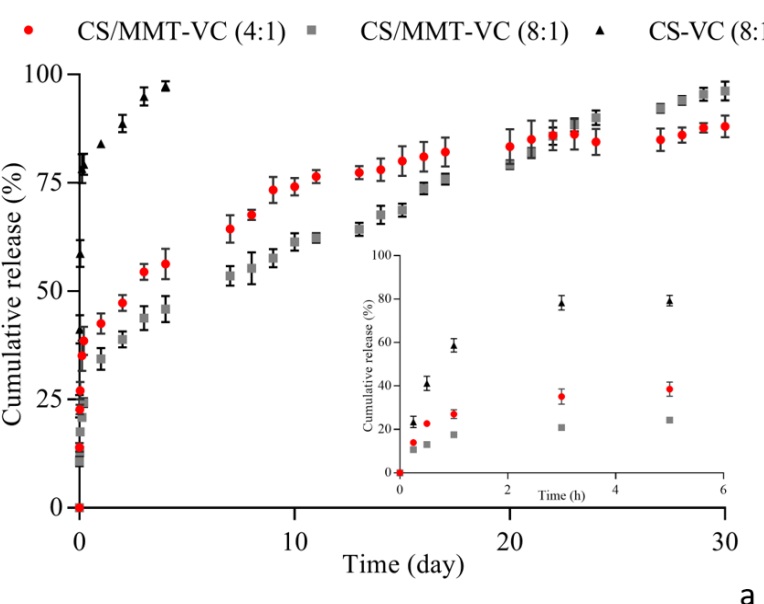

- CS/MMT-GC $(10: 1) \quad$ - CS/MMT-GC (20:1) \ CS-GC (20:1)

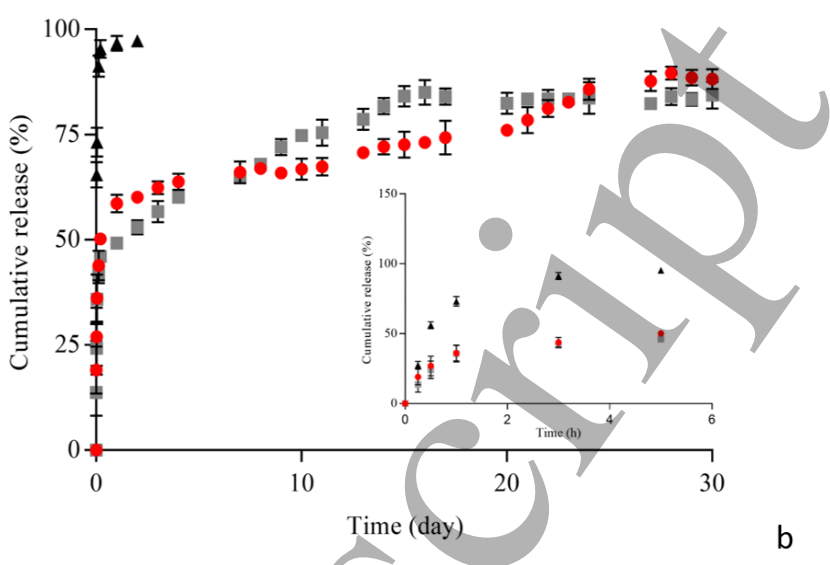

Figure 10. The cumulative release of Vancomycin (a) and Gentamicin (b) from CS and CS/MMT nanospheres

Mathematical relations corresponding the drug release during incubation were evaluated to predict the release rate at the specified time. Therefore, First-order, Higuchi, Korsmeyer-Peppas, Hixson-Crowell and Baker-Lonsdale empirical models were applied to determine the release kinetics and the dominant mechanism of Vancomycin and Gentamicin release from CS/MMT nanospheres. The initial porosity of the nanocomposite matrix was assumed negligible for Baker-Lonsdale model.

Mathematical models were applied to the experimental data and the release coefficients (k), Korsmeyer-Peppas release exponent $(n)$ and the correlation coefficients $\left(R^{2}\right)$ were depicted in Table 6 . The optimum model was selected according to the correlation coefficient values.

For Vancomycin-loaded nanospheres, it was found that the best fitting model is Higuchi based on the data presented in Table 6. The model claims the release of Vancomycin from nanocomposite matrix is a time-dependent process based on Fickian diffusion where the drug reservoir does not dissolve during the release ${ }^{31}$.

For Gentamicin loaded groups, the best model was found to be Baker-Lonsdale, the model developed from Higuchi especially for spherical drug carrier systems ${ }^{32}$.

Furthermore, first $60 \%$ of drug release data were fitted to Korsmeyer-Peppas model and " $n$ " value was used to characterize the release mechanism. It was found that each system has "n" value below 0.45 indicating that Fickian diffusion is the main mechanism in drug delivery from spherical CS/MMT nanocomposites ${ }^{33}$. In literature, chitosan based particulate nanocomposite systems followed Fickian diffusion mechanism for release of Repaglinide which shows diffusion of dissolved drug across the matrix that mechanically enhanced with cellulose nanocrystals ${ }^{34}$.
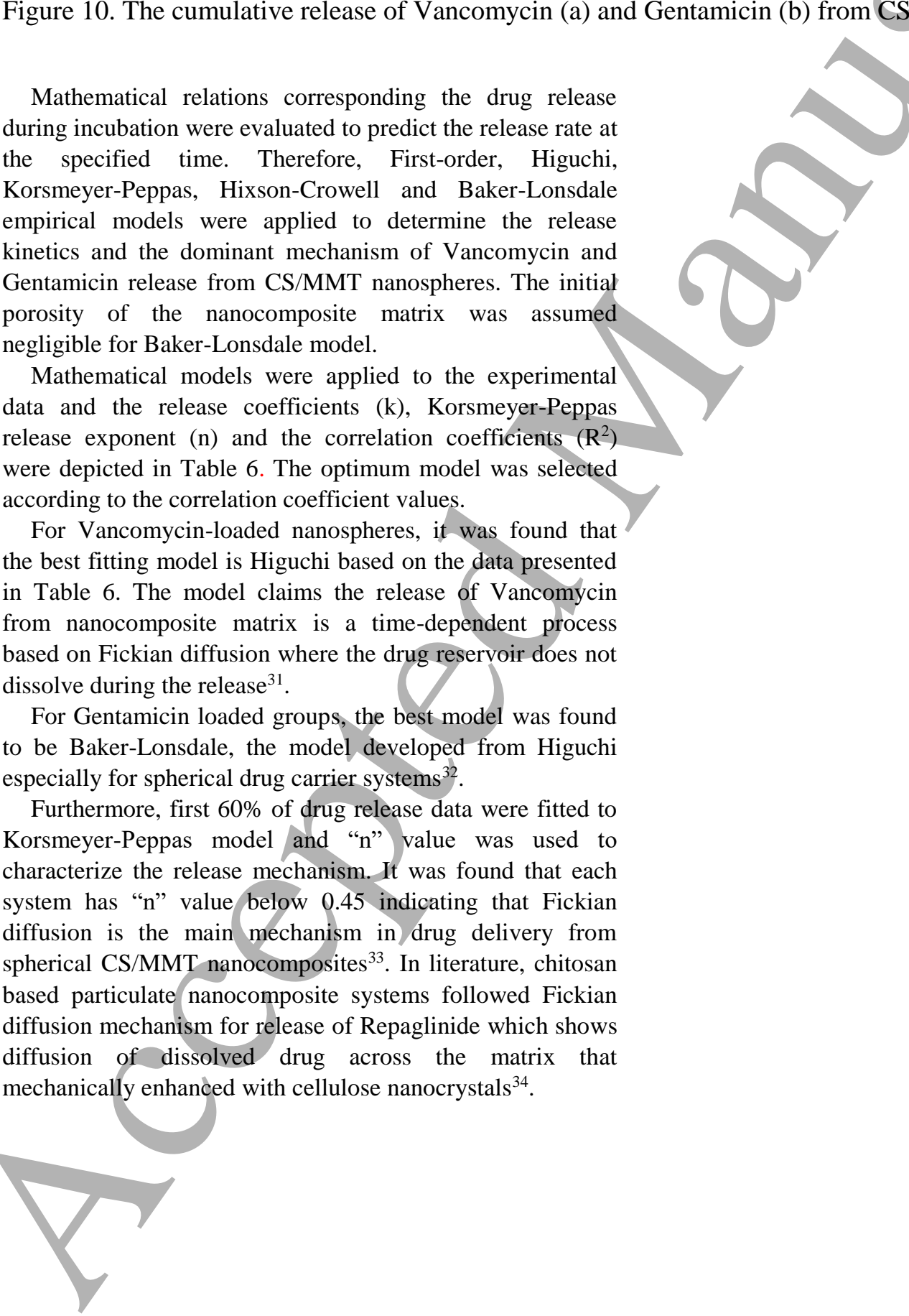
Table 5. Release kinetic coefficients for CS/MMT nanospheres.

\begin{tabular}{|c|c|c|c|c|c|}
\hline \multirow{2}{*}{ Models } & \multirow{2}{*}{} & \multicolumn{2}{|c|}{ Vancomycin } & \multicolumn{2}{c|}{ Gentamicin } \\
\cline { 2 - 6 } & & $\mathbf{4 : 1}$ & $\mathbf{8 : 1}$ & $\mathbf{1 0 : 1}$ & $\mathbf{2 0 : 1}$ \\
\hline \multirow{2}{*}{ First order } & $\mathbf{K}$ & 0.003 & 0.004 & 0.002 & 0.002 \\
\cline { 2 - 6 } & $\mathbf{R}^{\mathbf{2}}$ & 0.909 & 0.954 & 0.809 & 0.806 \\
\hline \multirow{2}{*}{ Higuchi } & $\mathbf{k}$ & 2.470 & 3.037 & 1.935 & 2.170 \\
\cline { 2 - 6 } & $\mathbf{R}^{\mathbf{2}}$ & 0.935 & 0.992 & 0.885 & 0.878 \\
\hline \multirow{2}{*}{ Hixson-Crowell } & $\mathbf{K}$ & -0.007 & -0.004 & -0.002 & -0.002 \\
\cline { 2 - 6 } & $\mathbf{R}^{\mathbf{2}}$ & 0.859 & 0.979 & 0.853 & 0.767 \\
\hline \multirow{2}{*}{ Baker-Lonsdale } & $\mathbf{R}^{\mathbf{2}}$ & 0.931 & 0.962 & 0.938 & 0.833 \\
\hline \multirow{2}{*}{ Korsmeyer-Peppas } & $\mathbf{n}$ & 0.193 & 0.238 & 0.215 & 0.191 \\
\cline { 2 - 6 } & $\mathbf{R}^{\mathbf{2}}$ & 0.922 & 0.996 & 0.952 & 0.802 \\
\hline
\end{tabular}

Table 6. Effect of in vitro release media (6 h, 24 h, 25 days) against $E$. coli and $S$. aureus

\begin{tabular}{|c|c|c|c|c|c|c|}
\hline & \multicolumn{3}{|c|}{ Inhibition zone of $E$. coli $(\mathrm{mm})$} & \multicolumn{3}{|c|}{ Inhibition zone of $S$. aureus (mm) } \\
\hline Groups & 6 hours & 24 hours & & 6 hours & 24 hours & 25 days \\
\hline $\begin{array}{c}\text { (+) control } 1 \\
(\text { Amoxycylin) }\end{array}$ & \multicolumn{3}{|c|}{$10 \pm 0.00$} & \multicolumn{3}{|c|}{$6 \pm 0.00$} \\
\hline $\begin{array}{l}\text { (+) control } 2 \\
\text { (Vancomycin) }\end{array}$ & \multicolumn{3}{|c|}{$5 \pm 0.00$} & \multicolumn{3}{|c|}{$11.3 \pm 0.01$} \\
\hline (-) control (Blank) & \multicolumn{3}{|c|}{ 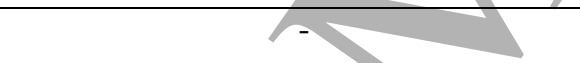 } & \multicolumn{3}{|c|}{-} \\
\hline CS/MMT-VC (4:1) & $14 \pm 0.00$ & $11.3 \pm 0.01$ & $10.0 \pm 0.01$ & $7.9 \pm 0.01$ & $5.3 \pm 0.03$ & $5.3 \pm 0.03$ \\
\hline CS/MMT-VC (8:1) & $14.7 \pm 0.01$ & $10.7 \pm 0.01$ & $9 \pm 0.00$ & $5.5 \pm 0.01$ & $4.8 \pm 0.02$ & $4.4 \pm 0.02$ \\
\hline CS/MMT-GC (10:1) & $14.7 \pm 0.02$ & $10.0 \pm 0.01$ & $10.3 \pm 0.01$ & $2.8 \pm 0.01$ & $4 \pm 0.00$ & $3.1 \pm 0.01$ \\
\hline CS/MMT-GC (20:1) & $11.7 \pm 0.02$ & $9.7 \pm 0.01$ & $8.8 \pm 0.00$ & $3.8 \pm 0.01$ & $4.1 \pm 0.01$ & $3.8 \pm 0.01$ \\
\hline
\end{tabular}

\subsection{Antimicrobial activity}

To confirm the relevance of the in vitro release study, the antimicrobial activity of the release media was investigated for Gram-positive $S$. aureus and Gram-negative E. coli. The minimum inhibition concentration of selected antibiotics on selected bacteria were determined with serial dilution tests (Figure 11). According to results, $0.25 \mathrm{mg} / \mathrm{L}$ of Vancomycin and Gentamicin was enough to inhibit bacteria colonization. On the other hand, $S$. aureus colonization was prevented with $2 \mathrm{mg} / \mathrm{L}$ of Gentamicin or $0.75 \mathrm{mg} / \mathrm{L}$ of Vancomycin. The results showed that released drug concentrations in all time intervals were higher than MIC values ensuring the antimicrobial activity. Therefore, it could be claimed that the therapeutic drug level can be achieved with the developed nanocomposite system without exceeding the toxic concentration.

Inhibition zones were clearly observed on discs diffusing the release media of nanospheres taken at specific time intervals ( 6 hours, 24 hours and 25 days). In negative control (blank disc), no inhibition zone was observed. Effect of released drugs against $E$. coli and $S$. aureus were observed as clear zones after 24 hours of incubation depicted in Figure 12. The inhibition zones were given in Table 7 .

It can be observed that the antimicrobial activity against $S$. Aureus was slightly increased with the increase in drug 
concentration which may be due to the increased drug penetration to bacterial cell wall. In addition, the inhibition zone at the $6 \mathrm{~h}$ period was larger, which is attributed to the burst release in short-time period. Similarly, Ryu et al. (2014) studied the effect of release kinetics on the antimicrobial activity of Gentamicin releasing PCL microspheres against $S$. aureus and E. coli. It was indicated that larger inhibition zones were achieved in the groups having burst drug release ${ }^{35}$. Similarly, Ambrogi et al. (2017) observed that MMT incorporation in chitosan based films
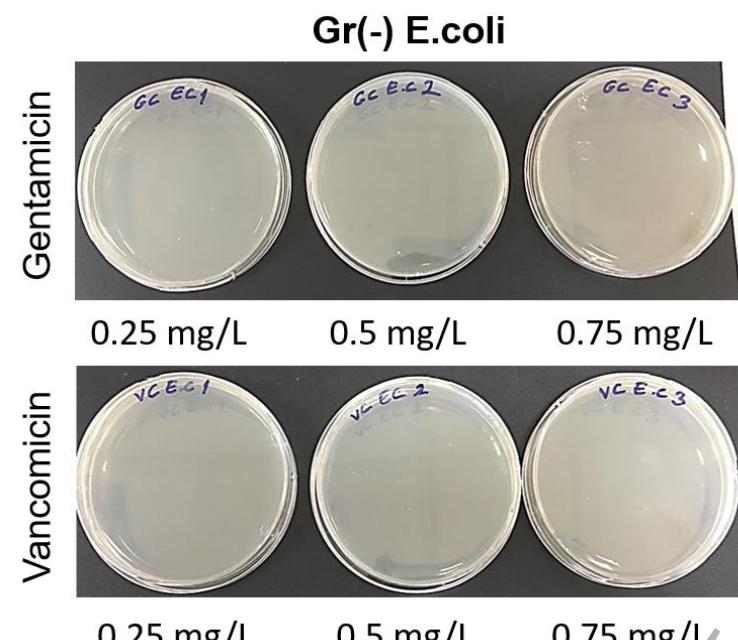

Figure 11. Minimum Inhibitory Concentration (MIC) determination of Vancomycin Hydrochloride and Gentamicin Sulphate by serial dilution test. caused slightly lower antimicrobial activity due to slower antibiotic release caused by intercalation of drugs into clay layers $^{36}$.

During the antimicrobial activity tests, optimum conditions $(\mathrm{pH}$, medium composition and temperature) for the proliferation of the test microorganisms were supplied. Consequently, higher antimicrobial activity is expected in the real case since pure strains cultured in laboratory conditions show maximum growth.
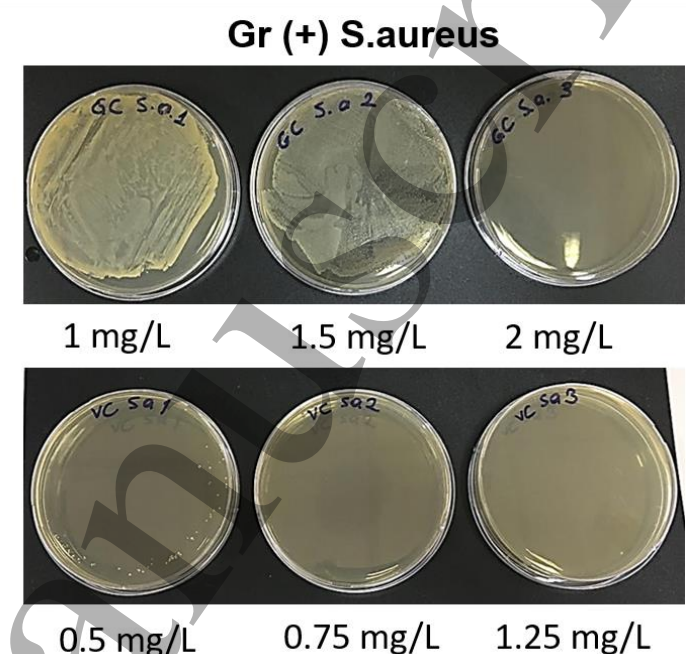

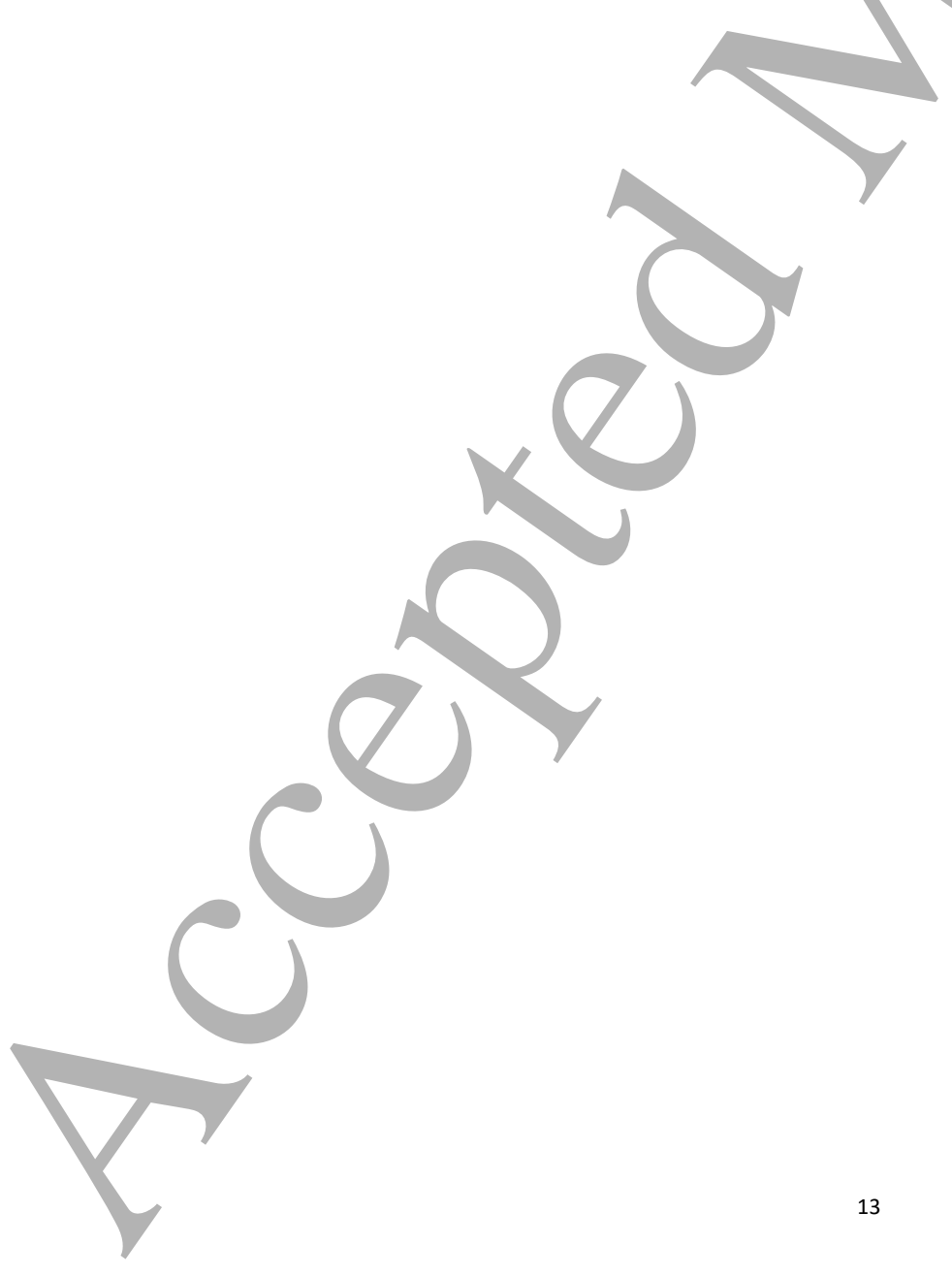




\section{E.coli}
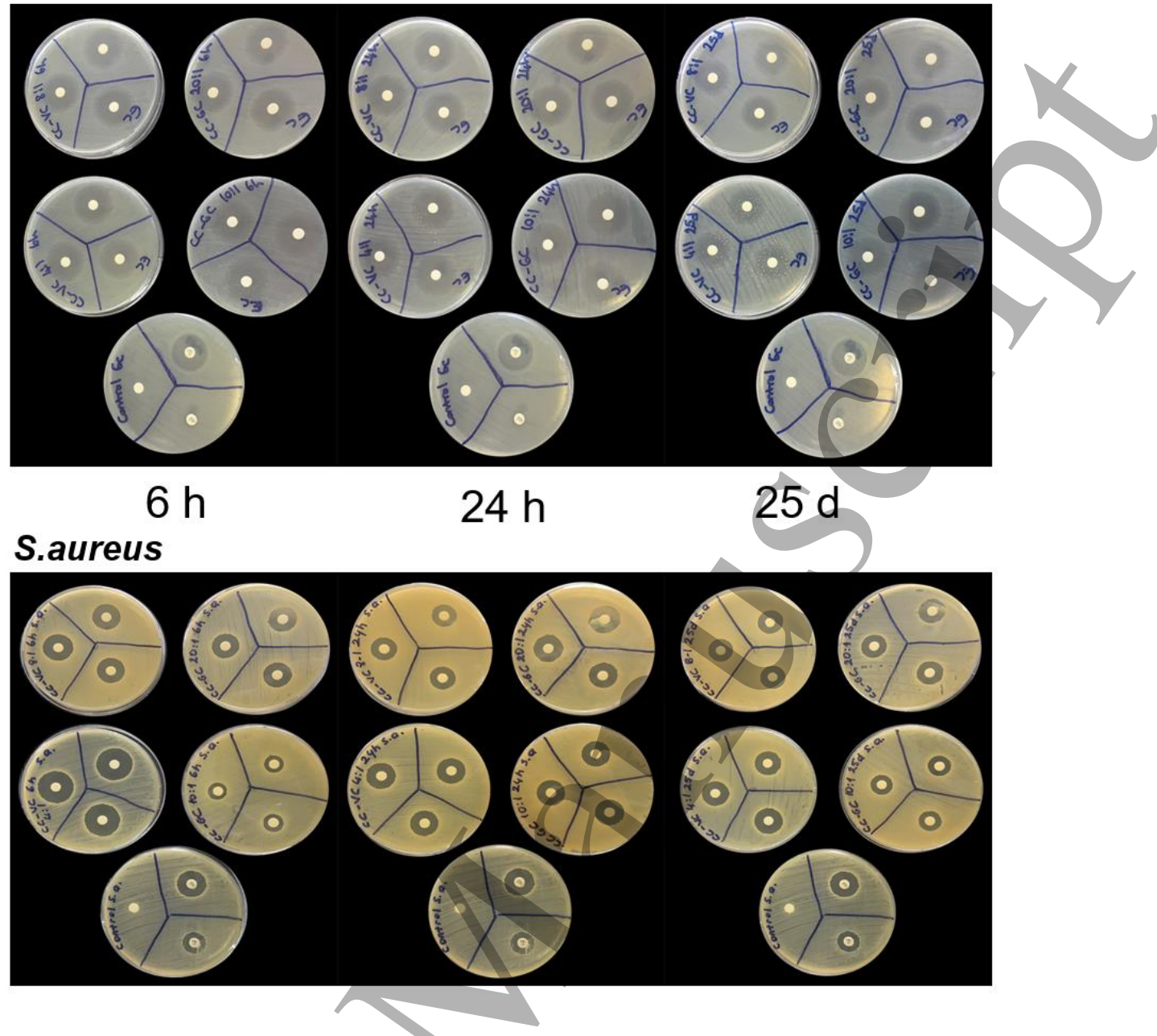

Figure 12. Effect of release media against E. coli and S. Aureus at incubation times of $6 \mathrm{~h}, 24 \mathrm{~h}$ and 25 days.

\subsection{In vitro cytotoxicity}

Cytotoxicity of the nanospheres on NIH/3T3 and SaOS-2 cell lines was evaluated by $24 \mathrm{~h}$ extraction of nanospheres in 24, 48 and 72 hours of incubation and depicted in Figure 13(a,b) respectively. It was observed that nanospheres did not cause any cytotoxic effect on cells. Indeed, all groups showed good viability (>95\%). Statistically, drug loaded nanospheres showed significant viability increase in incubation time for both cell line $(\mathrm{P}<0.05)$. In some groups, non-significant viability decrease at 72 hours of incubation was observed according to the possible contact inhibition of cells. Previous studies showed that CS/MMT composites do not show any cytotoxic behavior also to Caco-2 cells and rat stem cells ${ }^{37,38}$. 

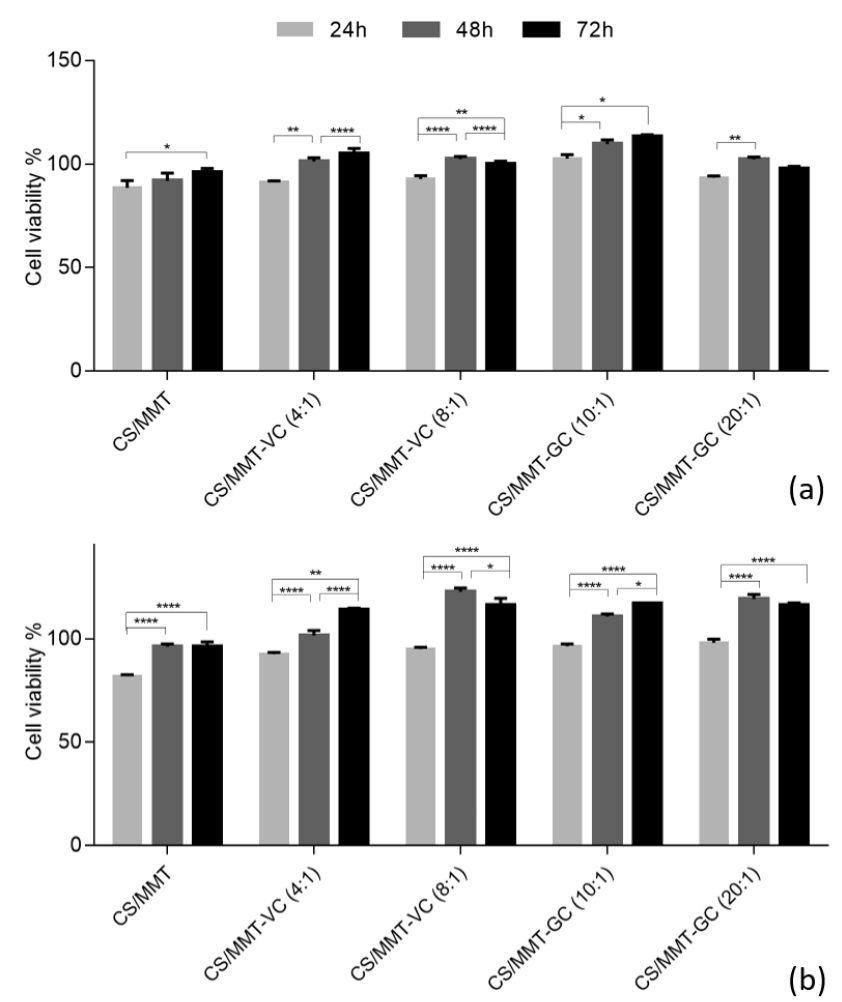

(b)

Figure 13. In vitro cytotoxicity of nanospheres against NIH/3T3 (a) and SaOS-2 (b) cell lines

\section{Discussion}

Infection is one of the most serious drawbacks of bone implantation operations which can cause necrosis of the tissue. Therefore, two weeks of antibiotic treatment is recommended in general as a precaution to eliminate the risk of possible infections ${ }^{39}$. Sustained antibiotic delivery is needed at the implant site to guarantee the prevention from microbial contamination of the implant.

One of the most challenging point in long-term drug delivery applications is to ensure the stability of the carrier during the treatment period and to ensure that it is degraded into non-toxic monomers after it releases the cargo. Besides its peculiar properties over other polymers for drug delivery applications, the poor stability of chitosan restricts its practical applicability in long-term release systems ${ }^{40}$. Nanocomposite systems can be considered as a solution to overcome disadvantages of biopolymer-based systems as well as improving mechanical and barrier properties even at very low concentrations compared to conventional composites that needs 40-50 wt. \% fillers ${ }^{41}$. Recently, polymer-montmorillonite (MMT) nanocomposites have been used in pharmaceutical applications to improve drug bioavailability by changing the release rate or distribution of cargo in carrier matrix ${ }^{42}$. This control over release profile can minimize the side effects of drugs, such as toxicity, and provides a therapeutic concentrations of drug in treatment period $^{36}$. In this study, fabricated CS/MMT nanospheres combines several advantages to achieve controlled antibiotic release at the defect media. The exfoliated nanocomposite structure prolongs the stability of drug carrier in physiological conditions since it serves a tortuous pathway to the drug for diffusion. MMT nanosheets can act as a crosslinker to ensure stability and slower release rate of hydrophilic drugs. The hydrophilic and cationic nature of the chitosan facilitates intercalated/exfoliated CS/MMT nanocomposite structure by cyclic application of highpressure homogenizer.

After nanocomposite formation, it was aimed to achieve nano-scale particles with spherical shape and smaller size. Morphology and the homogenity in the particle size are critical parameters for drug carrier materials to predict the release pattern more precisely. In this study, one-step electrospraying was selected as a production method to achieve higher yield compared to other production techniques. However, the disability of size control during operation yield us to make an experimental design to find the effect of process variables on nanosphere size. According to results, a voltage of $20 \mathrm{kV}$, distance of $10 \mathrm{~cm}$ and a flowrate of $10 \mathrm{~mL} / \mathrm{h}$ was selected as optimum conditions to achieve smaller nanospheres with uniform size. A mathematical model was derived with a high regression coefficient (0.96) which can be further used to achieve nanocarriers with a desired size. Afterwards, characterizations were performed to observe the morphology and the hydrodynamic size distributions of the drug loaded nanospheres. Experimental results point out that the decrease in polymer to drug ratio (P:D) leads to formation of larger particles. The increase in nanosphere size can be attributed to the drug integration in the polymer matrix. Literature findings also support that drugs incorporation in polymer matrix increase the size of the carriers when compared to the neat carrier material ${ }^{37}$. These results were found in good agreement with Vancomycin loaded CS microspheres and Gentamicin loaded CS nanoparticles in literature ${ }^{43,27}$.

The zeta potential of the carrier material gives an insight about the stability of the system and has a major influence on release profile and circulation in the body fluid. The stability of the carrier material in a surrounding fluid is an important criterion to overcome the aggregate formation in nanoparticulate drug delivery systems. The stability of the nanoparticles was guaranteed by electrostatic interactions between CS and MMT since they show opposite charges in physiological conditions.

Studies revealed that drug encapsulation efficiency strongly depends on the particle size, concentration of the active agent and the processing method ${ }^{44,45}$. According to the results, it could be concluded that the entrapment efficiency of the CS/MMT matrix was improved with the increase in 
polymer to drug ratio (P:D). Prepared nanospheres showed high encapsulation efficiency (76-95\%) which increases the applicability of the system. Therefore, the low efficiency of conventional drug administration methods could be overcome with presented carrier system entrapping the drug with a high efficiency and sustaining the release over time required for antibiotic treatments. The incorporation of the drug into the polymer matrix becomes easier as P:D increases, which results in higher entrapment efficiency value.

The most important criterion for drug delivery system would be to supply therapeutic level of drug during treatment. Therefore, here we demonstrated that CS/MMT nanocomposites sustained the drug release for weeks whereas bare chitosan nanospheres were degraded in hours. MMT nanoclay acted as a barrier in the polymer matrix to decelerate the drug diffusion rate. This significant difference in release periods can be attributed to interactions between silicate layers with drugs and chitosan with Coulombic interactions, van der Waals fore and $\mathrm{H}$ - bonding 5 .

In first 6 hours, burst release profile was observed due to possible diffusion of drug molecules adsorbed on nanosphere surface. This profile can be beneficial since most of the local infections after surgery occurs at this post-implantation period $^{46}$. However, it is not enough to protect the defect region against bacterial inflammation. Studies indicated that biofilm formation can be seen at the tissue-material interface if long-term drug release cannot be achieved ${ }^{47}$. Therefore, initial burst release was followed by 30 days of sustained release period which was desired in the post-implantation period $^{48}$. The developed two-step release model that diffuses drug in a controlled manner can be beneficial to overcome the local infection, then the gradual release of antibiotics prevents the bacterial recolonization.

In chronic osteomyelitis, mostly described bacterial isolates are Staphylococcus species and Gram-negative bacteria. S. aureus is known as an important bone pathogen that causes bone destruction and failure in orthopedic implants ${ }^{49}$. In addition, it was reported that total knee replacement operations can result in a bacterial infection caused by $E$. $\operatorname{coli}^{50}$. Local Vancomycin and Gentamicin release is selectively used in bone infection cases for both $\operatorname{Gr}(+)$ and $\operatorname{Gr}(-)$ bacteria. It is known that intercalation of antibiotics into clay layers does not change the antimicrobial activity $^{51}$. In the presented system, it was indicated that released Gentamicin and Vancomycin locally affected both pathogens which shows the availability of the prepared nanospheres as effective drug therapy. CS/MMT composite itself might promote the antibacterial activity with its bactericidal effect against $E$. $\operatorname{coli}^{52}$.

Besides its strong antibacterial properties, in vitro cytotoxicity analysis suggested that fabricated CS/MMT nanospheres are cytocompatible with fibroblast and human osteoblast-like cell lines, which makes them an ideal candidate for antibiotic delivery agents in bone infection treatment.

\section{Conclusion}

In this study, controlled antibiotic releasing chitosan/montmorillonite (CS/MMT) nanocomposite spheres were fabricated to improve antimicrobial efficacy while reducing the systemic toxicity of the antibiotics. MMT nanoclay was implicated in chitosan matrix/with microfluidizer homogenization to prolong the drug diffusion pathway and ensure the stability of the system. Drug loaded CS/MMT nanocomposites were obtained by electrospraying technique with a perfectly spherical shape in nano-scale. All groups obtain positive zeta potential due to the polycationic nature of chitosan. It was observed that drug entrapment capacity was increased by decreasing the loading amount. Initial burst release for 6 hours was achieved which is a critical period for bone infections. The drug release was proceeded in a controlled manner up to 30 days. It was found that Gentamicin and Vancomycin release from CS/MMT matrix were dominated by diffusion mechanism. In vitro release media of nanospheres showed strong antimicrobial activity against $E$. coli and $S$. aureus bacteria. In addition, fabricated CS/MMT nanocomposites did not show any cytotoxic effect against fibroblast and human osteoblast-like cells. The overall evaluations suggested that these antibiotic loaded CS/MMT nanospheres have a promising potential effect on bacterial infections at the post-implantation period. The antibiotic release from CS/MMT matrix can be controlled by alteration of polymer:drug ratio. Thus, high encapsulation efficiency and the sustained long-term release of these hydrophilic drugs make CS/MMT nanocomposite an ideal candidate to be used in nanoparticulate drug delivery systems.

\section{Funding}

This study was financially supported by The Scientific and Technological Research Council of Turkey (TUBITAK) under the name of Development of Controlled Antibiotic Releasing Chitosan/Silica Based Composite Scaffolds and Investigation of Their Potential for Hard Tissue Engineering Applications (1001-\#116M096).

\section{Ackowledgements}

The authors gratefully acknowledge Center for Materials Research (IZTECH CMR) for XRD, SEM and AFM analysis; Izmir Institute of Technology Biotechnology and Bioengineering Research and Application Center (IZTECH BIOMER) for NIH/3T3 cell line supply and antimicrobial activity study. The authors thank to Assist Prof. Dr. Meltem 
Alper from Aksaray University for supplying SaOS-2 cell line.

\section{References}

[1] Pacheco H, Vedantham K, Young A, Marriott I and El-Ghannam A 2014 Journal of Biomedical Materials Research Part A 102 4213-4223.

[2] Patel VR and Amiji MM 1996 Pharmaceutical Research 13 588-93.

[3] Anal AK and Stevens WF 2005 International journal of pharmaceutics 290 45-54.

[4] Noel SP, Courtney H, Bumgardner JD and Haggard WO 2008 Clinical orthopaedics and related research 4661377 1382.

[5] Depan D, Kumar AP and Singh RP 2009 Acta Biomaterialia 5 93-100.

[6] Yuan Q, Shah J, Hein SRDK and Misra RDK 2010 Acta biomaterialia 6 1140-1148.

[7] Salcedo I, Sandri G, Aguzzi C, Bonferoni C, Cerezo P, Sánchez-Espejo R and Viseras C 2014 Colloids and Surfaces B: Biointerfaces 117 441-448.

[8] Albalawi MA, Mohamed WS and Elsayed NH 2016 Der Pharma Chemica 8 27-34.

[9] Panagiotou T, Bernard JM and Mesite SV 2008 Nano Science and Technology Institute-Nanotech $139-42$.

[10] Khan A, Vu KD, Chauve G, Bouchard J, Riedl B and Lacroix M 2014 Cellulose 21 3457-3468.

[11] Gottipati R and Mishra S 2010 Chemical Engineering Journal 160 99-107.

[12] Geng X, Kwon OH and Jang J 2005 Biomaterials 265427 5432.

[13] Dash S, Murthy PN, Nath L and Chowdhury P 2010 Acta Pol Pharm 67 217-23.

[14] Hosseini SF, Zandi M, Rezaei M and Farahmandghavi F [42] 2013 Carbohydrate Polymers 95 50-56.

[15] Wang SF, Shen L, Tong YJ, Chen L, Phang IY, Lim PQ and Liu TX 2005 Polymer Degradation and Stability 90 123-131.

[16] Thakur G, Singh A and Singh I 2015 Scientia pharmaceutica 84 603-617.

[17] Shami Z, Sharifi-Sanjani N, Khanyghma B, Farjpour S and Fotouhi A 2014 RSC Advances 4 40892-40897.

[18] Cervantes-Uc JM, Cauich-Rodríguez JV, Vázquez-Torres H, Garfias-Mesías LF and Paul DR 2007 Thermochimica Acta 457 92-102.

[19] Abdollahi M, Rezaei M and Farzi G 2012 Journal of Food Engineering 111 343-350.

[20] Kosmulski M 2011 Journal of colloid and interface science 353 1-15.

[21] Satyanarayana KG 2004 Clay surfaces: fundamentals and applications (Vol. 1), Elsevier.

[22] Huang YC, Li RY, Chen JY and Chen JK 2016 Carbohydrate polymers 138 114-122.

[23] Honary S and Zàhir F 2013 Tropical Journal of Pharmaceutical Research 12 265-273.

[24] Yang CC, Lin CC, Liao JW and Yen SK 2013Materials Science and Engineering: C 33 2203-2212.

[25] Yao Q, Nooeaid P, Roether JA, Dong Y, Zhang Q and Boccaccini AR 2013 Ceramics International 39 7517-7522.
[26] Rapacz-Kmita A, Stodolak-Zych E, Ziabka M, Rozycka A and Dudek M 2015 Bulletin of Materials Science 38 10691078.

[27] Ji J, Hao S, Wu D, Huang R and Xu Y 2011 Carbohydrate polymers $\mathbf{8 5} 803-808$.

[28] Desai KGH and Park HJ 2005 Journal of microencapsulation 22 179-192.

[29] Ordikhani F, Dehghani M and Simchi A 2015 Journal of Materials Science: Materials in Medicine 26269.

[30] Selvig KA, Wikesjö UM, Bogle GC and Finkelman RD 1994 Journal of clinical periodontology 21 380-385.

[31] Higuchi T 1963 Journal of pharmaceutical sciences 52 1145-1149.

[32] Poletto FS, Jäger E, Ré MI, Guterres SS and Pohlmann AR 2007 International journal of pharmaceutics 345 70-80.

[33] Peppas NA and Colombo P 1997 Journal of Controlled Release 45 35-40.

[34] Abo-Elseoud WS, Hassan ML, Sabaa MW, Basha M, Hassan EA and Fadel SM 2018 International Journal of Biological Macromolecules 111 604-613.

[35] Ryu TK, Kim SE, Kim JH, Moon SK and Choi SW 2014 Journal of Bioactive and Compatible Polymers 29 445-457.

[36] Ambrogi V, Pietrella D, Nocchetti M, Casagrande S, Moretti V, De Marci S and Ricci M 2017 Journal of Colloid and Interface Science 491 265-272.

[37] Wang X, Du Y and Luo J 2008 Nanotechnology 19065707.

[38] Zhuang H, Zheng JP, Gao H and De Yao K 2007 Journal of Materials Science: Materials in Medicine 18 951-957.

[39] Dorati R, DeTrizio A, Modena T, Conti B, Benazzo F, Gastaldi G and Genta I 2017 Pharmaceuticals 1096.

[40] Szymańska E and Winnicka K 2015 Marine drugs 13 18191846

[41] Ali A and Ahmed S 2018 International Journal of Biological Macromolecules 109 273-286.

García-Villén F, Carazo E, Borrego-Sánchez A, SánchezEspejo R., Cerezo P, Viseras C and Aguzzi C 2019 Modified Clay and Zeolite Nanocomposite Materials Elsevier 129-166.

[43] Esmaeili F, Hosseini-Nasr M, Rad-Malekshahi M, Samadi N, Atyabi F and Dinarvand R 2007 Nanomedicine: Nanotechnology, Biology and Medicine 3 161-167.

[44] Cevher E, Orhan Z, Mülazımoğlu L, Şensoy D, Alper M, Yildiz A and Özsoy Y 2006 International journal of pharmaceutics 317 127-135.

[45] Cerchiara T, Abruzzo A, Di Cagno M, Bigucci F, BauerBrandl A, Parolin C, Vitali B, Galucci MC and Luppi B 2015 European Journal of Pharmaceutics and Biopharmaceutics 92, 112-119.

[46] Rumian $\mathrm{E}$, Tiainen H, Cibor U, Krok-Borkowicz M, Brzychczy-Włoch M, Haugen HJ and Pamuła E 2016 Materials Science and Engineering: C 69 856-864.

[47] Ordikhani F, Tamjid E and Simchi A 2014 Materials Science and Engineering: C 41 240-248.

[48] Rao N and Lipsky BA 2007 Drugs 67 195-214.

[49] Kanellakopoulou K and Giamarellos-Bourboulis EJ 2000 Drugs 59 1223-1232.

[50] Dixon P, Parish EN and Cross MJ 2004 The Journal of bone and joint surgery 86 39-42. 
[51] Ghadiri M, Hau H, Chrzanowski W, Agus H and Rohanizadeh R 2013 RSC Advances 3 20193-20201.

[52] Lertsutthiwong P, Noomun K, Khunthon S and Limpanart S 2012 Progress in Natural Science: Materials International 22 502-508. 